$10-7-2021$

\title{
Adolescent girls and COVID-19: Mapping the evidence on interventions
}

\author{
Sarah Blake \\ Population Council \\ Miriam Temin \\ Population Council \\ Tara Abularrage \\ Nihal Said
}

Follow this and additional works at: https://knowledgecommons.popcouncil.org/departments_sbsr-pgy

Part of the Family, Life Course, and Society Commons, and the Gender Equity in Education Commons How does access to this work benefit you? Let us know!

\section{Recommended Citation}

Blake, Sarah, Miriam Temin, Tara Abularrage, and Nihal Said. 2021. "Adolescent girls and COVID-19: Mapping the evidence on interventions." New York: Population Council, under the auspices of The Adolescent Girls Investment Plan (AGIP). 
Adolescent Girls Investment Plan

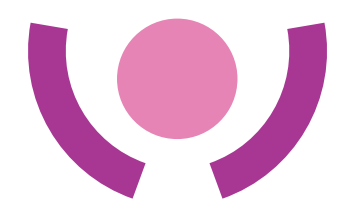

population COUNCIL

Ideas. Evidence. Impact.

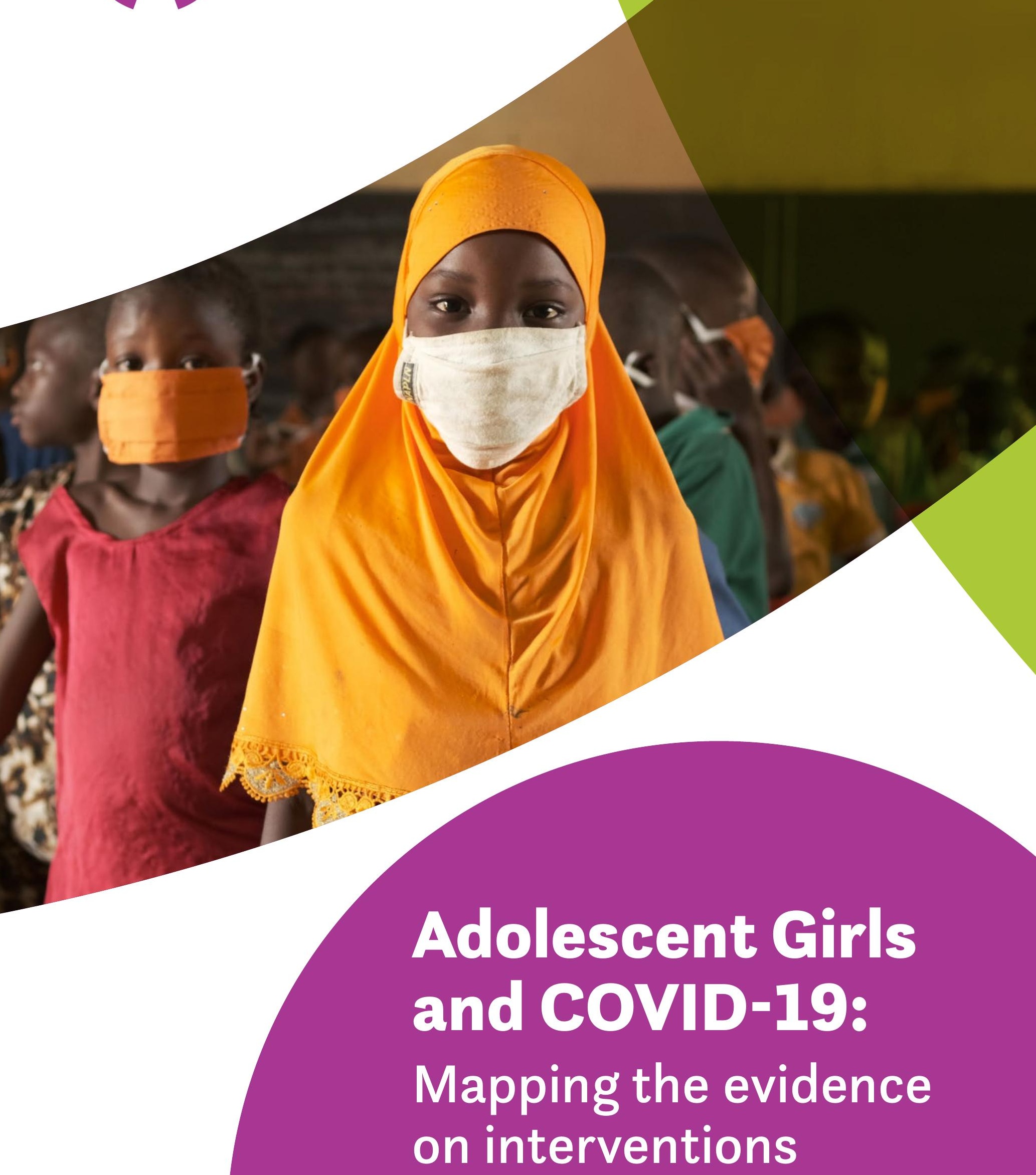




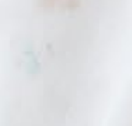




\section{Contents}

1 Synopsis

2 Introduction

3 Summary of Evidence Review Methodology

Evidence Gap Map Construction

$4 \quad$ Findings

Intervention Characteristics

Outcomes by Domains

Study Design Details

5 Implications: Responding to Research Questions

Summary recommendations to reduce COVID-19 related risk and increase opportunities for adolescent girls in low- and middle-income settings

6 Annexes

Annex I: COVID-19 and adolescent girls pathways: Summary of hypothesized risks and opportunities

Annex II: Evidence review methodology

Annex III: References 

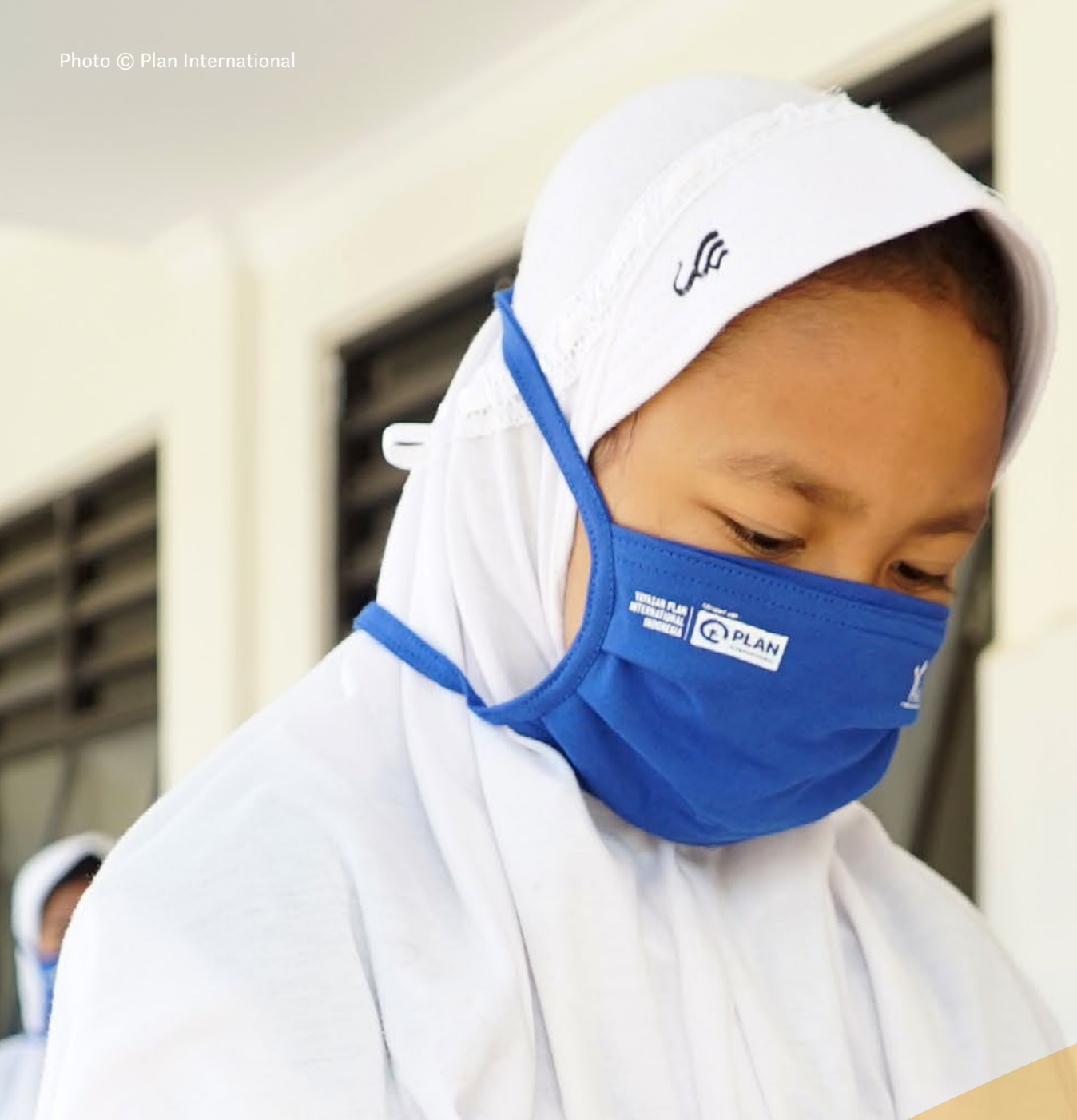


\section{Synopsis}

With the COVID-19 crisis continuing to evolve, evidence on the effectiveness of short-term emergency-oriented responses and long-term mitigation strategies is expanding but still limited. There are, and will continue to be, substantial evidence gaps on programming to address risk across outcomes of importance to adolescent girls. More evidence is needed to slow the risks posed by the pandemic for this sub-population, which can help guide gender- and age-responsive prevention and impact mitigation investments. Evidence from approaches delivered in other unstable contexts may offer important lessons for decision-making in the current context. Recognizing this, the Population Council conducted a structured review of existing evidence collected prior to the pandemic, across low- and middle-income country contexts (under the auspices of the Adolescent Girls Investment Plan, AGIP1). The review aimed to advance four goals, which are addressed in the summary below and in detail through the report:

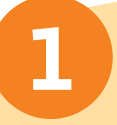

Identify high-potential intervention approaches that contribute to reducing girls' risks of contracting COVID-19, including interventions intended to promote individual girls' uptake of protective behaviors such as Information, Education and Communication (IEC) messages and personal protective equipment (PPE) distribution.

Identify short-term emergency interventions that hold promise to mitigate the secondary effects of COVID-19 on girls in contexts where recurrent outbreaks and control measures disrupt health and social services, schooling, or community-based programs.
Identify longer-term developmental intervention approaches that hold promise for "building back better," with and for adolescent girls in the longterm recovery period, once disease transmission has been controlled or stabilized globally but economic or social effects remain. These include measures that address long-term effects, and those that relate to critical elements of physical or social infrastructure.

Identify priority evidence gaps by assessing the concentration of evidence that exists on: the types of interventions that have been evaluated; the outcomes that evaluations have prioritized; the girl sub-populations that evaluations include; and the forms of instability or exclusion that intervention or evaluation designs describe.

1 The Adolescent Girls Investment Plan (AGIP) is a global partnership co-chaired by Plan International and Girls Not Brides (GNB) that aims to stimulate the development of investment frameworks and tools for decision-makers to confidently deliver a comprehensive approach to advance gender equality through girl centred approaches and actions. AGIP members include Plan International, Girls Not Brides (GNB), International Planned Parenthood Federation (IPPF), Gender and Adolescence: Global Evidence (GAGE/ODI), Population Council, International Center for Research on Women (ICRW), Malala Fund, and Women Deliver. Other technical supporters and partners include Coalition for Adolescent Girls, Bill and Melinda Gates Foundation (BMGF), Ford Foundation, She Decides, and Save the Children. 
The results of the review are summarized in an Evidence Gap Map (EGM). The EGM shows the concentration of evidence on specific interventions that were evaluated for their effects on outcomes among adolescent girls. It does not describe whether the interventions were effective in influencing those outcomes-i.e., if they were successful or not. Instead, it describes the number of times the outcomes have been measured across the available evidence base.

Based on the size of the evidence base on various interventions as portrayed in the EGM, as well as complementary information extracted from evaluation reports on the strength of the evidence, we identified promising interventions and pressing evidence gaps to inform future research priorities for reducing adolescent girls' risks and enhancing their opportunities.

This report describes the results of the review of evaluation evidence and their implications, accompanied by illustrative extracts of the EGM itself.
While understanding the quantity of evaluation evidence is useful to advance goals such as informing research priorities and identifying welldocumented interventions, it also has limitations. For instance, in contrast to systematic reviews or meta-analyses, our review does not culminate in clear conclusions regarding the relative effectiveness of different interventions against a range of outcomes. We recommend "promising" interventions based on the concentration of evidence on their effects combined with supplementary knowledge on the strength of the evidence, recognizing that more research is needed to establish them as "successful." For these reasons, our conclusions point to the need to accompany programmatic investments with more evaluation.

\section{1: Evidence Gap Map Structure ${ }^{2}$}

The EGM describes the quantity of reported effects...

\section{of these INTERVENTION CLUSTERS:}
A. Cash or asset transfer
B. Mass information and supply distribution
C. School-based or formal educational interventions
D. Institution-based exclusive of schools
E. Built environment
F. Community-based services/interventions
G. Complementary interventions

\section{on these OUTCOME DOMAINS:}
A. Gender empowerment
B. Health (includes general health, infectious disease, $\mathrm{SRH}$, mental health)
C. Education
D. Livelihoods
E. Violence and child marriage
F. Water, sanitation \& hygiene (WASH)
G. Civic participation

2 The Evidence Gap map is available here. Read more on how to interpret the Evidence Gap Map here. AGIP is grateful to zieimpact.org for providing open access to their platform to develop this map. 


\section{Summary of Findings: Recommendations to Reduce COVID-19 Related Risk and Increase Opportunities for Adolescent Girls in Low- and Middle-Income Settings}

\section{To Reduce Immediate/Acute COVID-19 Infection Risks}

To effectively reduce adolescent girls' direct infection risk, multiple approaches to health education must be combined with efforts to address structural factors.

- Lessons from distribution of IEC materials, school supplies and study materials, menstrual health management (MHM) supplies, and other tangible goods directly to girls offer relevant lessons for delivery approaches for girls. For example, resources should be distributed directly to girls and/ or caregivers to increase the likelihood of reaching girls, and products and messaging should be tailored to the context and girl "segment" to ensure access and relevance.

Girls' uptake of protective practices is not guaranteed even if resources are distributed to households. Collecting sex- and agedisaggregated data on distribution, receipt, and control over resources and/or cash is vital to track whether and how household resources distributed are shared among household members.

\section{To Mitigate Secondary Effects}

Evidence is evolving regarding the types and duration of the pandemic's secondary effects, while study results to inform interventions to mitigate the secondary effects of COVID-19 on adolescent girls, such as school closures, remain limited. Existing evidence suggests that inclusive, gender responsive planning that balances the benefits of restrictions (e.g., on movement) with risks (e.g., girls' school drop-out) is essential (UNICEF, 2021).

Emerging evidence on education during COVID-19-related school closures suggests that plans should explicitly focus on adolescent girls as schools open and close, offering different modalities that respond to their circumstances and needs. For instance, students want active engagement with school staff to sustain learning (e.g., safely exchanging materials with teachers)(UNICEF, 2021).
Responsive cash and asset transfers can contribute to offsetting risks resulting from household economic strains. For example, transfers can support girls to stay in school or remain unmarried, although critical questions remain, such as the level at which transfers can influence behavior (the threshold effects) and whether transfers should be conditional, labeled, or unconditional. It is essential to intentionally support and track coverage to ensure the intended beneficiary subpopulations are not overlooked, including directing targeted support measures to marginalized communities, households, and individuals.

The pandemic has underscored the need to prioritize "learning while doing" interventions to address urgent challenges exacerbated by the pandemic, including violence prevention and response, access to sexual and reproductive health services, and social isolation, stress, and household conflict.

\section{To Build Back Better}

The opportunity to "build back better" post-pandemic may offer a window to promote a gender-transformative vision for broader change. Ensuring that social services respond to adolescent girls in all their diversity is part of this ambitious vision. Tracking gender- and age-specific effects of recovery approaches has the potential to help make policies and programs more gender-transformative.

Interventions that incorporate emergency measures, such as cash or asset transfers that can be integrated into social protection systems, have an important place in recovery strategies. "Cash-Plus" combination approaches appear to be especially valuable, while community-based girls' groups may offer an important and otherwise missing platform for girls to meet safely and restore valued relationships with mentors and peers. 
School-based health, gender, and violence prevention education; youth-friendly health services; and protection and legal services are promising intervention areas that are under-funded and under-studied. Tailoring interventions, for example by offering remote and clinic-based services, may increase service use among diverse sub-populations. Lessons about flexible service delivery models necessitated by the pandemic may have long-term benefits for access by marginalized populations.

- Multilevel interventions that combine household-level economic investments, such as cash transfers and/or savings accounts, and/or social interventions such as structured community dialogues on gender and girls' education, also are promising.

\section{To Fill Critical Evidence Gaps}

The review extracted evidence that suggests promising areas for investment at different stages of the pandemic, especially the mid- to long-term recovery. Overall, cash transfers and community-based girls' group programs hold promise for adolescent girls. Other potential investments include education and health services that are responsive to adolescent girls' particular circumstances. Community or householdlevel action to address harmful gender norms, for instance related to girls' schooling and GBV, also are likely to benefit adolescent girls. Tailoring programming to girls' heterogeneity and layering multi-sectoral action are helpful approaches.
However, many critical evidence gaps limit policymakers' and implementers' ability to support adolescent girls as the COVID-19 pandemic evolves. The review turned up little to no evidence on effective approaches to address several outcome domains:

- Interventions with particular salience to ongoing pandemic outbreaks, such as access to and use of PPE, supplies, or practices related to handwashing and social distancing did not appear in our searches.

- We found no evaluation evidence on the effects of common emergency measures on girls under 18 including cash transfers, food and supply distribution, hotlines, emergency services for girls experiencing household violence, and emergency health services.

- No studies explored questions related to girls' access to essential technologies, such as mobile phones, nor how to ensure girls' access to safe transportation.

In the recovery phase, more evidence is needed to inform programming to link girls with gender-transformative social protection, health, education, financial, and other services. This should include implementation science, which can increase understanding of the role of contextual factors. This evidence is vital to interpreting evaluation results and understanding what works for whom in what settings. Nuanced findings that reflect adolescent girls' heterogeneity also are needed to disentangle the unique features of individual beneficiaries, study contexts, and conditions. 


\section{Introduction}

The COVID-19 pandemic is both an enduring global health emergency and a set of overlapping social and economic crises unfolding at different paces, with different impacts, over time and across contexts. The content and timing of government responses, existing structural conditions, and community and individual practices mean that both the severity of COVID-19-specific risks and secondary dimensions of the crisis vary. Common COVID-19 control measures, such as travel restrictions and business and school closures, reduce the threat of COVID-19 transmission while they also can exacerbate other threats to health, safety, and wellbeing (Abdullah et al., 2010; Banati et al., 2020; UNFPA, 2020; Youssef \& Jones, 2020). Overall, disparities in primary and secondary pandemic effects within and between countries are exacerbating existing inequality and threatening progress in areas such as gender equity in education and women's economic status (UNFPA, 2020; Pinchoff et al., 2020). Recognizing these challenges, multilateral agencies, governments, and non-governmental organizations (NGOs) have expanded social protection measures, such as cash transfers, food distribution and nutrition assistance; introduced computer, mobile phone, television and radio-based alternatives to schooling; and expanded measures such as hotlines that offer support for gender-based violence survivors (Gentilini et al., 2020; Matovu et al., 2021; van Zandvoort et al., 2020).

While adolescent girls are among the target groups for alternative services and risk mitigation efforts, this is no guarantee that they have adequate support. Children, and girls in particular, often are overlooked in efforts to plan for emergencies (IRC $\&$ VOICE, n.d.). Pre-pandemic, many girl-serving programs and services were already underresourced, and the pandemic has exacerbated this situation as critical interventions have been deprioritized or suspended (Amin et al., 2020; Banati et al., 2020; Bellerose et al., 2020; UNFPA, 2020; Youssef \& Jones, 2020).

The adolescent-specific effects of COVID-19 threaten boys and girls in different ways, filtered through gender norms, roles, and structural forces. Evidence on the pandemic's effects on adolescent girls suggests that across settings, girls may encounter exclusion and barriers to access for basic services and support on one hand, and greater responsibilities at home on the other, jeopardizing learning gains and school attendance (Akmal, Hares, et al., 2020; Amin et al., 2020; Baird et al., n.d.; Banati et al., 2020; Bellerose et al., 2020; Youssef \& Jones, 2020). Though effects of the pandemic and associated mitigation measures vary widely by context, emerging evidence suggests that enduring school closures and household economic shocks have created acute and potentially expanding risks for girls' health and safety. These effects may concentrate among girls who are already in precarious situations. For example, where substantial economic barriers to girls' education already exist, long-term school closures may constitute a 'last straw', driving girls permanently out of school (Bellerose et al., 2020; Jones et al., 2020; Youssef \& Jones, 2020). Further emerging evidence suggests that even within communities with high levels of economic instability, girls' and young women's experiences of the pandemic differ by age. For example, the economic effects of the crisis, combined with inadequate or inaccessible learning alternatives, may leave older girls at higher risk of school dropout than younger girls (Bellerose et al., 2020). Social isolation, household economic stress and crowding, and worries about the future may further jeopardize girls' health and well-being (Jones et al., 2020; Baird et al., 2020) .

Pre-pandemic conditions and emerging evidence on the pandemic response indicate that critical structural factors limit the amount and type of investments for adolescent girls. For example, woman-led civil society organizations, including those representing girls and their interests, tend to receive limited funding, constraining their ability to hold decision-makers accountable (OECD, 2016). And measures introduced under the guise of a response to COVID-19 may also carry destructive ideological dimensions; for example, many countries initially misclassified reproductive health care as "non-essential," imposing new barriers to service access (IPPF, n.d.). 
Recognizing the pandemic's role in exacerbating existing inequality and undoing progress on gender equity, members of AGIP's Evidence Sub-Committee ${ }^{3}$ developed an Adolescent Girls and COVID-19 Conceptual Framework (see Annex I). The framework describes pathways that describe the pandemic's hypothesized risks and opportunities for adolescent girls (AGs). The aim of the framework is to foster understanding of how multiple dimensions of impact-across sectors - may play out at the intersection of female gender and young age to inform research and investment. The framework identifies near, medium, and longer-term opportunities to respond to risk with actions that target girls and young women directly, and that ensure community-wide activities are gender- and ageresponsive so girls are not left out.

\section{COVID-19 Pathways for Adolescent Girls: Hypothesized Risks and Opportunities by Outcomes}

\begin{tabular}{|c|c|c|}
\hline Domain & AG Risks (illustrative) & AG opportunities (illustrative) \\
\hline 1. Health & $\begin{array}{l}\text { Curtailed access to SRH services } \\
\text { esp. STI treatment }\end{array}$ & $\begin{array}{l}\text { Greater prioritization of community } \\
\text { health worker cadres }\end{array}$ \\
\hline 2. Education & $\begin{array}{l}\text { Limited access to alternative } \\
\text { learning/remote schooling }\end{array}$ & $\begin{array}{l}\text { Expansion of quality non-formal } \\
\text { schooling }\end{array}$ \\
\hline 3. Livelihoods & $\begin{array}{l}\text { Over-burdened with household } \\
\text { reproductive labor }\end{array}$ & $\begin{array}{l}\text { Employment for new cadres of } \\
\text { community-based workers }\end{array}$ \\
\hline 4. Violence & $\begin{array}{l}\text { Breakdown of social cohesion } \\
\text { restricts girls' mobility }\end{array}$ & Increased access to violence hotlines \\
\hline $\begin{array}{l}\text { 5. Water \& } \\
\text { Sanitation }\end{array}$ & $\begin{array}{l}\text { Decreased water supply, poor } \\
\text { hygiene at home/water points }\end{array}$ & $\begin{array}{l}\text { Young women as handwashing } \\
\text { educators/ promoters }\end{array}$ \\
\hline 6. Transport & $\begin{array}{l}\text { Reduced transportation alternatives } \\
\text { increase reliance on risky modes; } \\
\text { sexual violence }\end{array}$ & $\begin{array}{l}\text { Women-run transportation } \\
\text { companies }\end{array}$ \\
\hline $\begin{array}{l}\text { 7. Technology for } \\
\text { connectivity }\end{array}$ & $\begin{array}{l}\text { Limited means of mobile technology, } \\
\text { reliance on boys/men to access vital } \\
\text { information, social connections }\end{array}$ & $\begin{array}{l}\text { Innovations in gender-responsive } \\
\text { technology access and training }\end{array}$ \\
\hline
\end{tabular}

More evidence is urgently needed across the seven outcome domains described in the COVID-19 Pathways framework to slow the pandemic's detrimental effects on adolescent girls and reduce backsliding on the Sustainable Development Goals. In hopes of generating useful insights on promising approaches and priority research gaps, we reviewed evidence from past crises and unstable circumstances, and described what interventions have been evaluated against which outcomes. The results of the review are portrayed in an Evidence Gap Map on adolescent girls interventions in low-resource settings in the context of COVID-19 and extracted in Section 4 Findings. We hope that the analytical framework used to create the Gap Map also is relevant for assessing evidence on responses to other crises as they relate to adolescent girls. 


\section{Flow Diagram: Results of Literature Search}

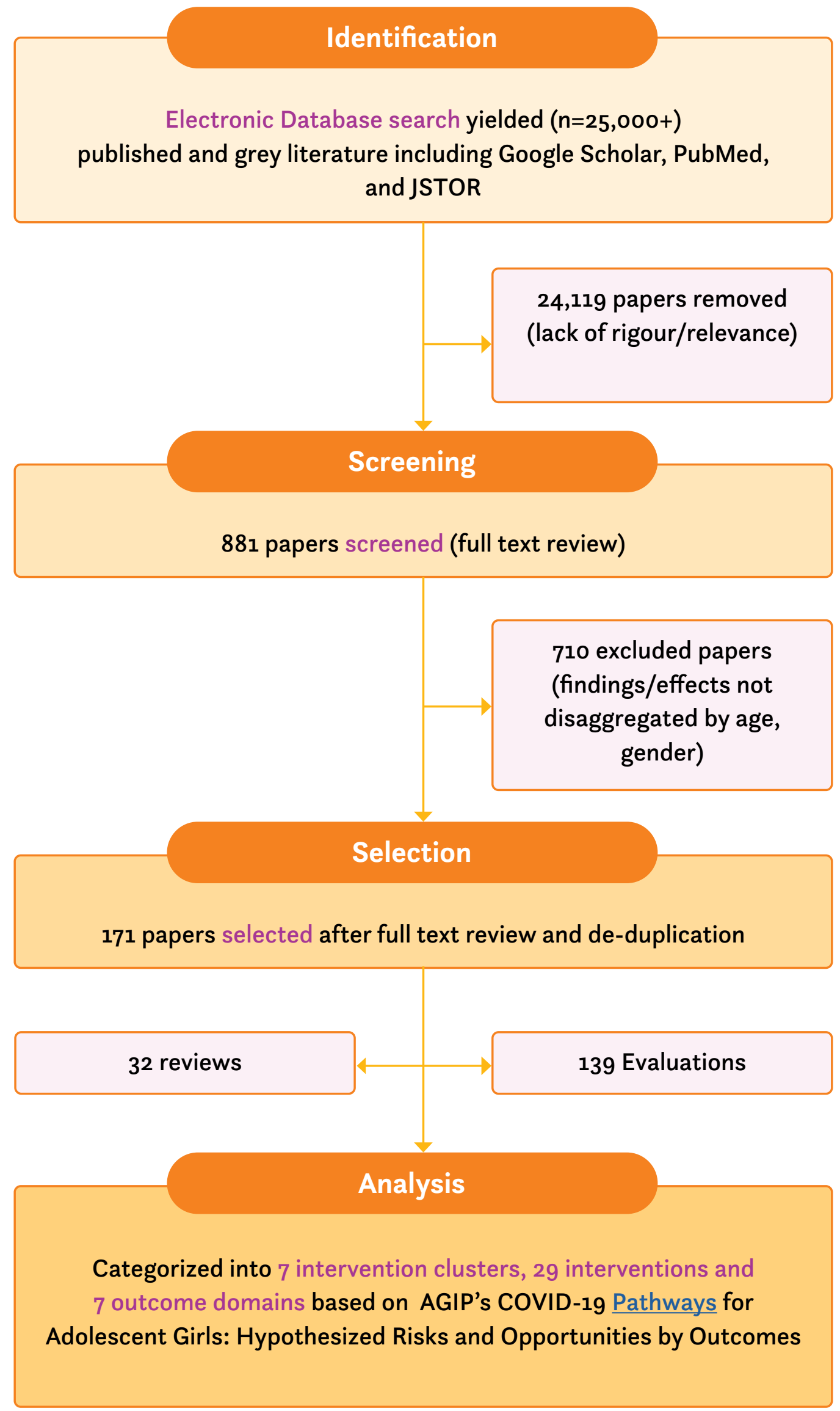




\section{Summary of Evidence Review Methodology}

We conducted a structured literature review centered on intervention approaches that engaged adolescent girls. The review methodology included an initial review of titles and abstracts, which enabled us to narrow the sample to reports of evaluations that stated they included adolescent girls. Along with single evaluations, we included systematic and other structured literature reviews of evidence on interventions that reached adolescent girls and reported girl-specific results. We only included qualitative, quantitative, and mixed methods evaluations and reviews that described the research methods and sampling criteria (see Annex II for methodology).

We conducted a full-text review of the evaluation and review reports that remained after the initial screening to verify fit with inclusion criteria. (see Figure 1) We extracted relevant details from the remaining reports, including:

- Intervention components: Organized by pre-defined categories and subcategories

- Methodology: Research method, rigor, sample size, and analytic approach and reported limitations

- Population: Description of girls included in study, including age and other sociodemographic information used in analysis; other populations studied

- Context: Region or regions, country, and community characteristics (rural, urban, peri-urban, camp), reference to emergency or instability, and type

- Outcome domains: Domains summarize the outcomes that were studied in the evaluations

In terms of the context of the evaluations, they occurred in one or more regions spanning: Asia (Central, East, South) and Pacific; Middle East and North Africa; Africa (East, West, Southern); and Latin America and Caribbean regions. The review included, but was not restricted to, evaluations in settings undergoing crisis, conflict, humanitarian or natural disasters, or economic shock. If instability or crisis of any kind was described, we noted the type of instability, such as infectious disease outbreak or climate-related disaster. ${ }^{4}$

We divided the sample of 171 publications into two categories: first, reviews that compiled findings from a number of different studies of a specific outcome (e.g., child marriage), intervention (e.g., youth-friendly health services), or sub-population (e.g., girls in urban settings); second, evaluations that used a variety of research methods to assess a single intervention, which ranged from small-scale pilots to large experiments as well as policy-based interventions implemented at scale.

\section{Evidence Gap Map Construction}

To advance the goals of the review, we organized the results of the evaluations into an Evidence Gap Map, which illustrates the scope and scale of available evidence according to interventions and broad outcome domains. Study results are described by intervention categories and subcategories. The Evidence Gap Map shows the amount of evidence available on the effects of interventions on specific outcome domains. The map is a visual representation of the quantity of reported effects of specific interventions on specific outcomes that are aggregated into outcome domains. To note, EGM is a description of the quantity of the reported effects within our sample, not what those effects are. The effects were not analyzed for the direction of change, size of change, nor are they restricted to statistically significant results or high-confidence reviews. Furthermore, it does not describe results that were not reported in publications nor effects that were not evaluated.

4 It was not possible to reflect this information in the EGM, however details are available on request. 
The bubbles in the map illustrate the concentration of the evidence (i.e., number of publications that reported effects of interventions on outcome domains), with grey bubbles representing all types of evaluations and orange bubbles representing all types of reviews. The size of the bubbles is proportionate to the number of publications that reported on the effects of interventions by outcome domains. The number of bubbles is larger than the total number of interventions or evaluations because many publications reported the effects of multiple interventions on multiple outcome domains; in addition, some evaluations generated multiple publications.

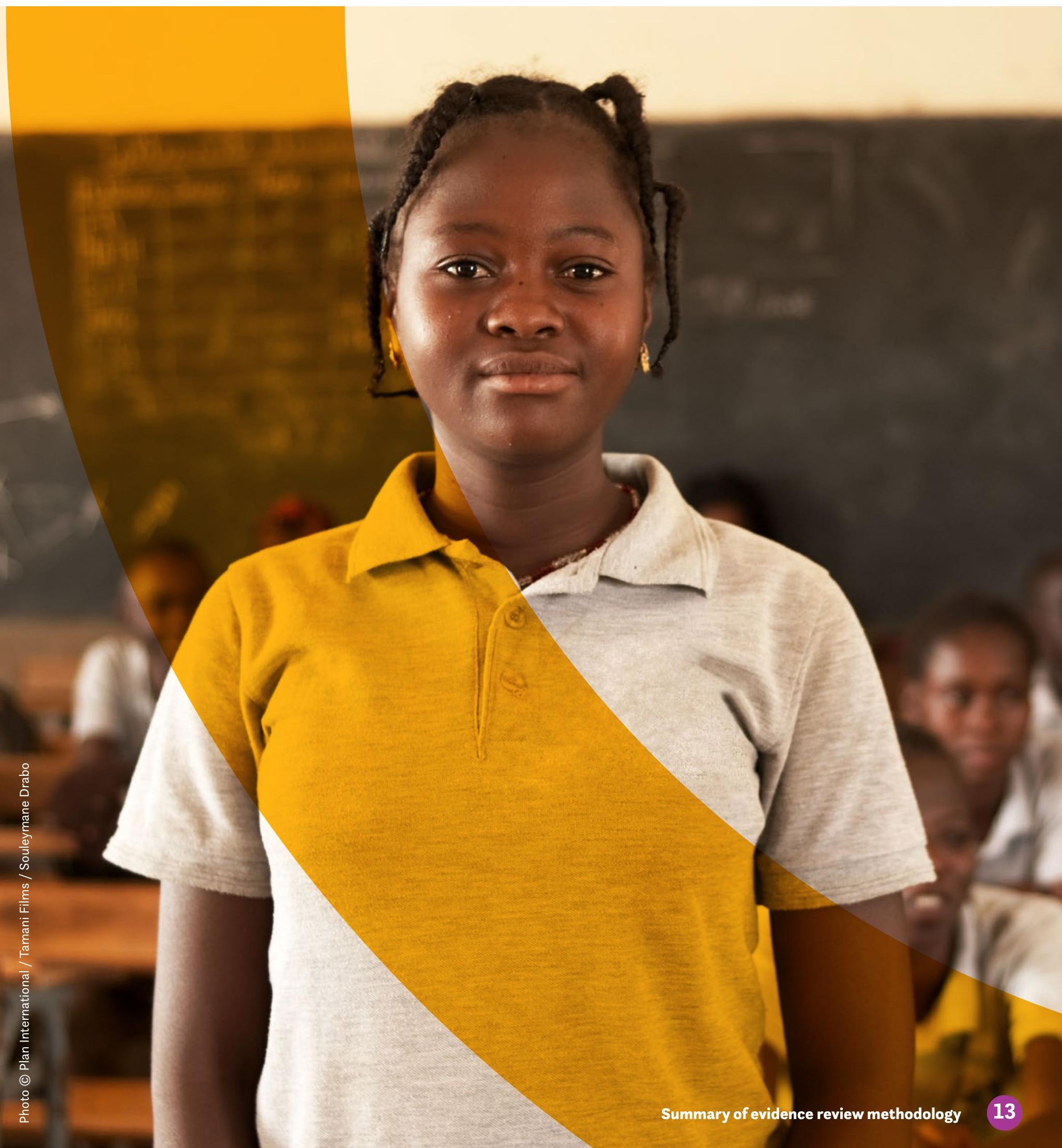




\section{Findings}

\section{Intervention Characteristics}

We grouped interventions into seven categories by intervention type (see Box 4.1). The categories are based on interventions' feasibility, application, and likely use under various stages of the COVID-19 pandemic and its response, which enabled us to draw conclusions from the results. The intervention categories are related to their relevance to phases of the pandemic onset, spread, and recovery, from flexible interventions that can meet urgent needs, with limited contact between interventions and participants, to those that are better suited to medium-to-long-term recovery. We identified subcategories within each intervention cluster as a basis for summarizing findings based on common types of program delivery approaches. A vast majority of studies evaluated multi-component programs that included interventions from multiple categories either in one study arm or across different sites and study arms.

In our sample, the intervention categories with the most results were: community-based approaches (specifically girls' groups), school-based interventions (specifically health education), and cash or asset transfers (specifically cash transfers). The subcategories include a variety of program models; for example, community-based girls' groups typically incorporated components such as savings accounts or complementary interventions with parents, boys, or community members. By contrast, nearly half of evaluations of cash or asset transfer programs included either a single intervention or compared variations in the same type of approach, such as conditional versus unconditional transfers. Other approaches, such as school-based health education, also reflected substantial variation in program models. Some approaches incorporated deliberate or stand-alone teacher training components, while others provided novel health education content as a package administered by external facilitators or teachers.

\section{1: Intervention categories*}

A. Cash/asset transfers including school fees

B. Low-contact services, mass education, or information dissemination

C. School-based or formal educational interventions

D. Non-school-based institutional interventions

E. Physical infrastructure

F. Community-based intervention

G. Complementary interventions

* Single evaluations often studied multicomponent programs that included interventions in different categories and/ or subcategories. In addition, similar interventions may appear in different intervention categories when they were implemented using different models and in different contexts, e.g., peer education in schools vs. in communities vs. youth centres.

\section{Category A: Cash and asset transfers, school fees}

\section{RESULTS}

The interventions in Category A encompass a range of approaches that can be administered at any phase of an emergency or in any context, which we grouped into three subcategories: cash, assets, and school fees. (see Box 4.2) Interventions in this category include direct transfers of money or assets selected for their equivalent value, such as livestock, to households or directly to girls. This category also includes school fees administered directly to schools. These were included because they operate with comparable mechanisms to other types of transfers in terms of having an inherent value and serving as an incentive. In general, cash and asset transfer programs pursued multiple objectives, such as promoting education, health, or livelihoods and economic activities and preventing child marriage. 


\section{2: Cash and Asset Transfer Interventions and Adolescent Girls}

Means-testing and targeting: Some cash transfer (CT) programs are means-tested, requiring participants to apply and prove membership in a designated economic status, such as having an income below a certain threshold. Many programs also target households with children belonging to a certain age category, with eligibility lasting until they reach a specific age. Some programs also focus on specific groups, such as orphans and vulnerable children, a group prioritized for HIV prevention interventions.

Recipients: Although the typical cash transfer recipients are adult caregivers, approaches that aim to benefit girls may transfer directly to girls themselves, to a girl and her caregiver, or to a caregiver.

\section{Conditioning}

- Conditional: Conditional cash or asset transfers are common among girl-specific approaches, either within national social protection schemes or within experimental approaches, with transfers conditioned on, for example, girls' school attendance or remaining unmarried for the duration of an intervention. Such measures require participating households or individuals to document that they meet the condition, although the level of enforcement of these requirements varies.

- Labeled: Labeled transfers, like conditional transfers, typically include a degree of means-testing and targeting. Such measures are "labeled" for use for schooling costs, for example, but do not include a requirement that recipients remain in school.

- Unconditional: Such approaches may be couched in a distinct purpose or aim but there is no requirement that recipients fulfill conditions.

School fee payment: Programs or social service providers transfer funds directly to a school for individual students, typically bypassing individual girls and their households.

Asset Designation: Where transfers are assets instead of cash, the nature of the in-kind transfer may be selected based on the asset's economic value or because of its suitability for a specific practice, for example, providing bicycles to girls based on the hypothesis that they would overcome economic and geographic barriers to schooling. (Muralidharan \& Prakash, 2017) Notably, school uniforms may be designated as "assets" or school supplies depending on the program's context, intervention, and objectives.

Timing and Repetition of Transfers: Transfer programs vary in terms of the amount of money or monetary value of the designated assets, as well as the timing, duration, and repetition of transfers. For example, programs may distribute transfers annually, often at the beginning of every school year, more frequently, or as a one-time benefit.

Visibility: Cash and asset transfer interventions vary in the venues and approaches used to present transfers to girls and/or families. Asset transfer programs that aim to benefit adolescent girls, in particular, may use high profile public events to raise the visibility of interventions and the value of girls' schooling or work.

\section{Category B: Mass information and supply distribution}

\section{RESULTS}

Category $B$ includes interventions that can be used to meet direct COVID-19 risks and/or address emergency or acute needs that may arise during a crisis; such approaches also may be used in later stages of an emergency. We distinguish them from other approaches because of their potential application under early-stage responses when economic activities, school, and other activities may halt.

The intervention subcategories in this cluster include mass media, social media, distribution of information, education, and communication (IEC) materials, and distribution of materials such as food, school supplies, or menstrual hygiene management (MHM) products. These varied in the delivery models and content. For example, measures that employ social media may rely on 
one-way or interactive two-way communications and may vary in their objectives, e.g., providing advice or distributing information. Supply distribution interventions may include one-time or repeated distributions. Notably, we did not find evidence of the effects on adolescent girls of community health promotion and communitybased distribution, which were included in this category.

Case study 1: Assessing the Effectiveness of a Text-Messaging Program on Adolescent Reproductive Health in Ghana (Rokicki, et al., 2017; Rokicki \& Fink, 2017)

Quantitative design (cluster-RCT) including 14-24-year-old urban girls \& young women

A mobile health platform used an interactive quiz game in which participants won airtime for texting correct answers to priority sexual $\&$ reproductive health $(\mathrm{SRH})$ questions. The program increased SRH knowledge; a higher level of engagement was associated with higher knowledge scores up to year later. Results were mixed for sexual behavior outcomes. Encouragingly, researchers found no evidence that the program disproportionally reached better-off groups.

\section{Category C: School-based or formal educational interventions}

\section{RESULTS}

Approaches in Category $\mathrm{C}$ are relevant to midterm and long-term recovery phases because they are feasible after pandemic-related restrictions begin to lift. This category includes interventions that use schools as platforms, such as school feeding programs. Evaluations of school-based interventions that included adolescent girls typically assessed the effects of educational content that fell outside of standard curricula, such as new or supplemental health or life skills content, anti-violence education, or financial literacy. A few studies included variations on these models, such as individual or group counseling or "school helper" programs, or interventions to train teachers or improve the school environment. Common features of Interventions in this category included: a reliance on schools to identify and recruit participants; engagement of school personnel in delivering interventions; and operations in school buildings. School-based peer education was a subcategory in this cluster. (see Box 4.3) 


\section{3: School-Based Health and Life Skills Education Models and Adolescent Girls}

Evaluations described variations in the design features of school-based interventions that may influence their feasibility and impact. These included:

Timing in relation to school day

- In-School: Studies may evaluate the delivery of existing curricular content or novel content or learning methods delivered during the school day as part of the regular course of lessons. These approaches typically target a specific grade level and aim to reach all members of a given cohort.

- After-school clubs: Clubs or groups that offer content outside of the school day may resemble community-based girls' groups in providing health and life skills content, a "safe space" to discuss concerns or express themselves, or participate in games and sports, and an older mentor. These approaches differ from formal school curriculum and community-based clubs in that they recruit participants directly from schools, and implement activities on school grounds.

Participants: Single-sex interventions vs. mixed-sex: Health education models may deliver content to mixed-sex groups; they also may target girls' schools or offer content in co-ed schools dividing girls and boys for the purposes of a given intervention, and/or recruit only girls.

Personnel: May include:

- Teachers: Teachers may be recruited as "resource" teachers who receive special training and support to deliver lessons to a defined cohort of students, or volunteer to lead after-school activities.

- Peer educators: Peer educators may include "peers" who are older and/or have completed more years of school than intervention participants, or they may be close in age. Peer educators may also lead activities or assist professionals.

- Trained specialists: Outside, non-school staff who implement interventions, and may include lay health educators, NGO staff, or other professionals

Content: Approaches may include health education content, including standard or official health education content such as abstinence-only or comprehensive sexual and reproductive health content; tailored supplemental content; or specialized learning in areas such as gender rights awareness, nonviolent communication, or financial literacy.

\section{Category D: Institutional interventions outside of schools}

\section{RESULTS}

Category $D$ includes interventions that use existing social and financial infrastructure, making them more viable in mid-term or long-term recovery. These interventions are delivered through institutions other than schools, such as health services and banking. This category includes savings accounts and other formal financial inclusion measures. Relevant health services may include static clinics and/or mobile clinics for HIV-related services. This category also includes formal vocational training, which resembles schooling but is delivered through different management structures. This category also includes other social services and interventions to promote access to them, along with youth centers and behavioral health or mental health interventions.

\section{Category E: Physical infrastructure}

\section{RESULTS}

Category E includes infrastructure interventions; our search included a broad set of infrastructure features relevant to adolescent girls, such as transportation. However, interventions in this category are rarely studied for their effects on individual girls. The only type of evidence on interventions in this category that fit our inclusion criteria related to water, sanitation, and hygiene (WASH), emphasizing school toilets and handwashing facilities. Physical upgrades to infrastructure, specifically for WASH, constitute an important component of "building back better" post-pandemic by upgrading physical facilities including in schools or community spaces. This is important for adolescent girls given their roles in domestic chores that rely on water, such as cooking and caregiving. 


\section{Category F: Community-based intervention}

\section{RESULTS}

Community-based interventions in Category $\mathrm{F}$ are more appropriate to mid-term mitigation or long-term recovery like institutional interventions. Community-based measures may be feasible when COVID-19-related restrictions on mobility and social gatherings ease because they typically incorporate intensive in-person engagement. They are distinguished from other interventions because they are separate from formal social protection infrastructure or services.

\section{4: Community-Based Girls Groups or "Safe Space" Programs and Adolescent Girls}

While many models of community-based programming for adolescent girls exist, community-based girls' groups, or "safe space" clubs, constitute a distinct model. To fit in this subcategory, interventions had to include regular meeting times for girls-only groups; structured health and life-skills learning content; a young adult female mentor.

These measures are different from schoolbased after-school programs because they recruit participants from a common geography, typically within walking distance of the meeting space, even if they focus on school-going girls and use schools as a meeting venue. As such, these approaches also are open to out-of-school girls who typically are excluded from schoolbased approaches. They also differ from both in-school and mixed-sex community-based approaches because the social and physical girl-only "safe space" is central to the model, providing girls a venue to build social ties with peer-age girls and mentors without concern of harassment or interference from boys.

Some community-based girls group include complementary components, such as financial literacy education, referral mechanisms for existing services, or organized sports activities, which we noted as complementary measures within the relevant subcategories.

Interventions in this category varied tremendously in their delivery models and implementation approaches. This category includes interventions that leverage existing community-based entities, such as savings groups, if they included adolescent girls. They also include mixed-sex, community-based groups for girls and boys meeting in the same settings, community-based girls' groups, or "safe space" clubs. (see Box 4.4) In addition, this cluster includes communitybased peer education programming.

\section{Category G: Complementary interventions}

\section{RESULTS}

Category $\mathrm{G}$ includes interventions that are more relevant to mid-term recovery or longterm transformative. It includes approaches that directly engage community members other than girls alongside interventions targeting girls, such as boys, young women, parents/caregivers, or community leaders. Subcategories in this category include: community mobilization or dialogue for adults and parallel or linked activities for caregivers of girls who participate in community-based or school-based interventions. In our sample, these design features of complementary interventions varied in implementation depth and structure, from single events to regular, weekly structured approaches.

Case study 2: Review of Girls' clubs, life skills programs \& girls' well-being outcomes. ODIGender \& Adolescence: Global Evidence (GAGE) (Marcus, et al., 2017)

Rigorous narrative review including 63 studies of 44 programs

This review synthesized empowerment effects of Girls' Clubs, Youth Development Clubs $\&$ life skills programs. It found substantial evidence of benefits of these programs on: social \& psychological empowerment, genderrelated attitudes, gender-discriminatory practices, knowledge of SRH \& legal rights, education \& civic participation. It further highlighted shortfalls of these programs in terms of populations reached (size, degree of vulnerability), sustainability (NGO vs. government-funded), scalability \& costeffectiveness. The authors observed that most programs studied were described as relatively short pilots or experiments, mostly reaching under 20,000 girls. 


\section{Outcomes by Domain}

\section{5: Outcome Domains}

1. Gender Equality and Girls' Empowerment

2. Health

3. Education

4. Livelihoods

5. Violence and Child Marriage

6. WASH

7. Other Outcomes

The evaluations in our sample measured a wide range of indicators that aligned to a range of sectoral outcomes. We grouped these into seven broad outcome domains (see Box 4.5) that roughly align with common development priorities and map to the theorized Pathways of COVID-19 for Adolescent Girls described above (details in Annex I). Each outcome domain encompasses both several levels of potential change, such as knowledge, attitudes, and practices that are along the causal pathway of changes in girls' status. Evaluations included a complex variety of measurement approaches, which we summarized in the broad outcome domains. For example, if an evaluation compared the effect of several interventions on health knowledge, behaviors, and outcomes, it appears in the Evidence Gap Map under "health" effects. If the same study included measures of impact on girls' attitudes towards gender inequitable social norms or self-efficacy, it also appears in the map under measures of "empowerment."

A vast majority of evaluations reported on outcomes in two or more domains. Evaluations of the most common intervention sub-categories-cash transfers, school-based health education, and community-based girls' groups-often measured outcomes related to health, education, and violence or child marriage prevention. For instance, it was common for school or community-based health or life skills education interventions to address topics such as gender equitable beliefs or girls' self-esteem in the gender and empowerment domain, alongside factual knowledge that may motivate girls to stay in school, prevent violence, or promote livelihoods. "Empowerment" outcomes typically were captured as intermediary variables for interventions that aimed to promote changes in health, education, or violence reduction. Evaluations typically collected data on livelihoods or financial literacy in the context of interventions to promote other outcomes, such as health, education, or child marriage prevention.
The health domain was the outcome domain measured most frequently. Following health, the most commonly measured outcomes domains were: violence and child marriage prevention, empowerment and gender equality or girls' empowerment, and livelihoods or economic status. We explain each outcome domain below.

\section{Gender Equality and Girls' Empowerment}

\section{RESULTS}

Case study 3: The Impact and Cost of

Child Marriage Prevention in Three African Countries (Erulkar et al., 2017)

Quasi-experimental design (cross-sectional population-based baseline/endline surveys) including 12-17-year-old in \& out-of-school rural girls in Ethiopia, Tanzania, Burkina Faso

This study aimed to identify a minimum package of interventions with the greatest possibility for scale-up to delay child marriage. It showed that simple, contextualized interventions can offer cost-effective strategies for delaying child marriage \& promoting girls' schooling. Approaches tested were: 1) community sensitization on social norms, 2) school supplies $\& /$ or fees for school return/continuation, 3) a conditional asset transfer (livestock) to girls \& families, 4) combination of the three approaches. Results were stratified by age $\&$ revealed varied effects by context \& age; younger girls participated more than older girls, while multiple approaches appeared beneficial for younger but not older girls' schooling.

Findings underscore the need to consider feasibility \& scalability in settings with high child marriage rates. Other important implementation considerations emerged:

- More complex models had lower coverage \& struggled with implementation quality;

- Social norm change approaches must be systematic \& intensive, not ad hoc;

- Conditional asset \& supply distribution must be frequent $\&$ make girls visible;

- School promotion efforts must consider available educational infrastructure. 
Gender equality and empowerment-related outcomes included the adoption of gender equitable attitudes, self-efficacy, and self-confidence. This domain also included strengthening of girls' social networks and other features of girls' individual "voice", or self-expression, and challenging of gender inequitable practices. These outcomes extended to girls' experiences of their households and communities, including expanding mobility and improving safety.

Program theories of change often position empowerment-related outcomes as intermediary steps towards changes in health, education, or violence prevention rather than an end in itself. A range of studies incorporated empowerment measures, however, many others did not measure this step along the causal pathway to changes in behavior or status. For example, evaluations of community-based girls' group activities typically included measures of girls' empowerment, while measures of the effects of school-based activities were less likely to do so even if both approaches aimed to promote healthy behaviors. Similarly, where cash and asset transfers appeared as standalone interventions, evaluators tended to assess their effects on outcomes like improving school retention or delaying marriage rather than girls' empowerment. By contrast, where transfers were combined with other intervention components, such as community-based girls' groups, measures of empowerment were typically included.

The evidence gap map extract below provides an illustration of the concentration of evidence of specific interventions on the Gender Empowerment outcome domain. The snapshot shows that 15 evaluations and 10 reviews of community mobilisation interventions reported effects on gender empowerment, whereas the effects of interventions such as boys' groups or male engagement are less studied for their Gender Empowerment effects.

\section{EGM Extract 1: Gender Empowerment and Complementary Interventions}

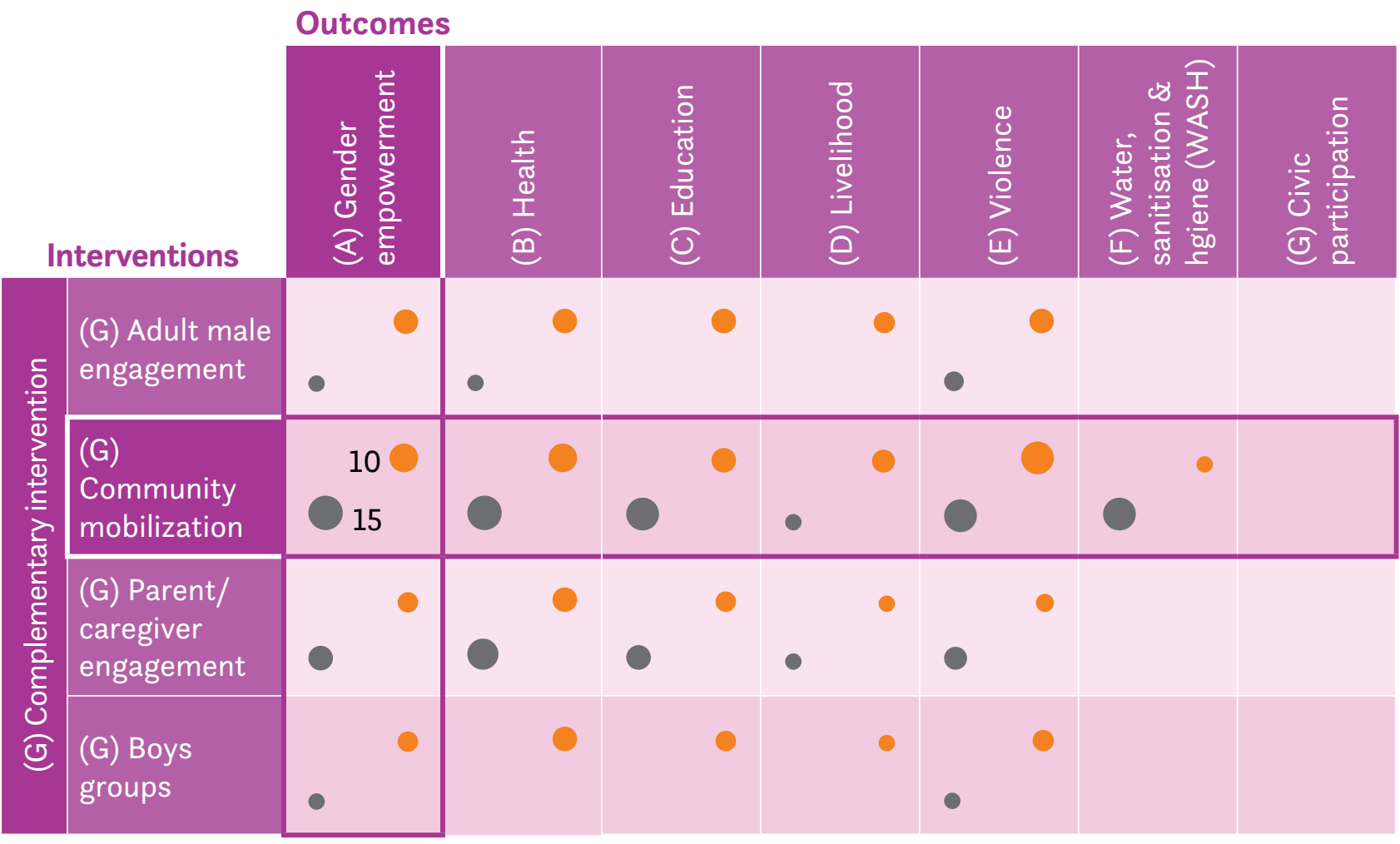

All types of evaluations All types of reviews 


\section{Health}

\section{RESULTS}

The health outcome domain includes knowledge, attitudes, and practices such as contraceptive or other reproductive health service use, delays in sexual debut or reductions in frequency of sex, and measures of health status such as HIV, herpes simplex virus infection, or unintended pregnancy. Along with sexual and reproductive health, other types of health outcomes include nutritional status, with a range of measures such as access to nutritious foods and dietary diversity, meal-skipping, variety in diet, and biological

measures deficiencies like anemia. Mental health, including depression or anxiety, falls into this category. It also includes general health status or use of primary health care, recent serious illness, or symptoms of infectious diseases, hospitalization, or specialized care.

The extract below shows that more evaluations (25) and reviews (17) studied the effects of life skills or health education on health-related outcomes than other types of interventions (e.g., $\mathrm{mHealth}$, only observed in 2 evaluations).

\section{EGM Extract 2: Heath and School-Based or Formal Educational Interventions}

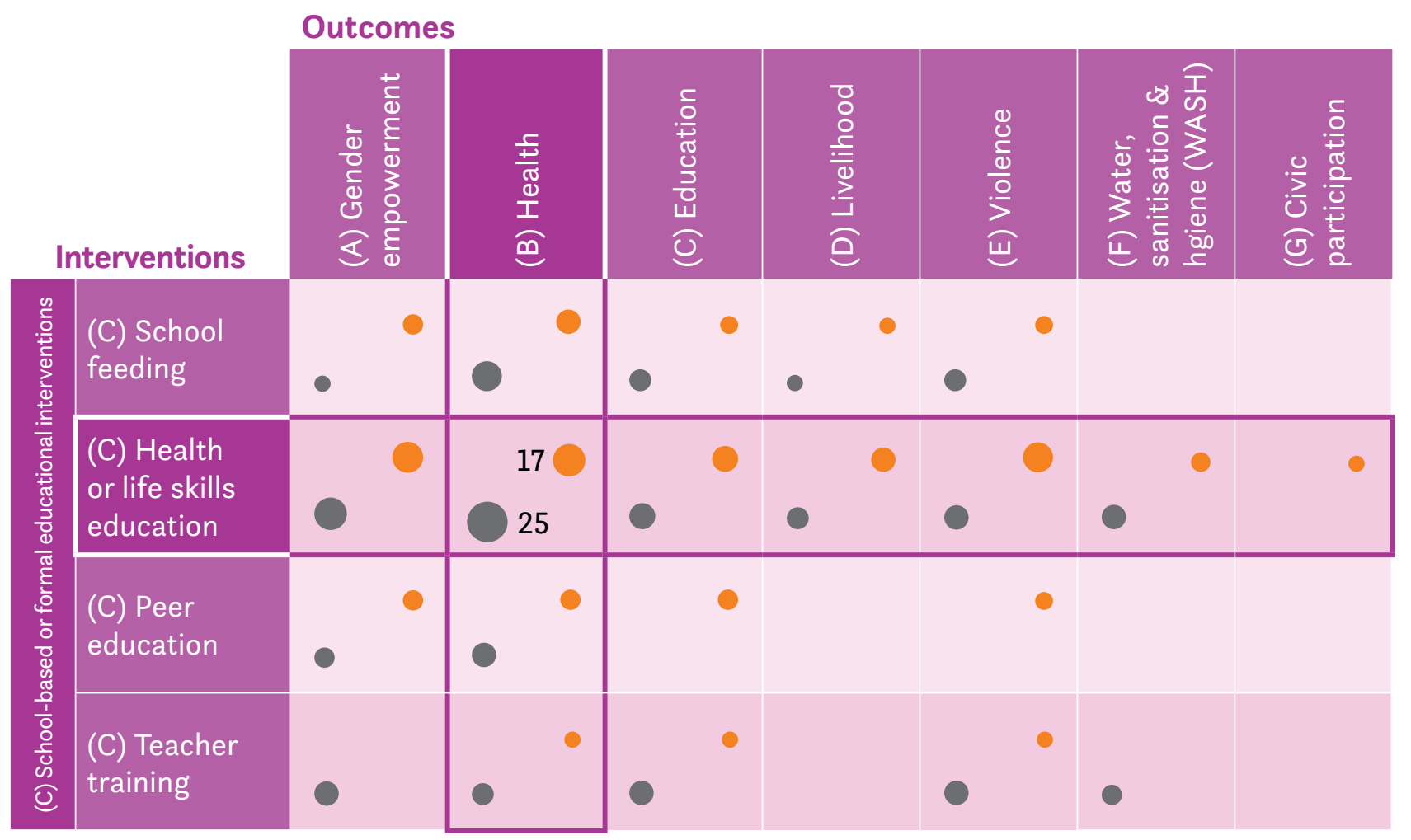

All types of evaluations All types of reviews 


\section{Education}

\section{RESULTS}

Education-related outcomes include school attendance, achievement, or progression along with learning outcomes. Education-related outcomes focus on girls' progression through school, often in relation to skills acquisition such as literacy and numeracy. These outcomes often were measured in relation to interventions such as cash or asset transfers and school

attendance and continuation, and/or to community-based girls' group programs.

The extract below shows that evaluations and reviews reported more often on the effects of cash transfers for girls' education than on the effects of other types of transfers: 29 evaluations and 8 reviews. fee payments to directly promote girls' school

\section{EGM Extract 3: Education and Cash or Asset Transfers}

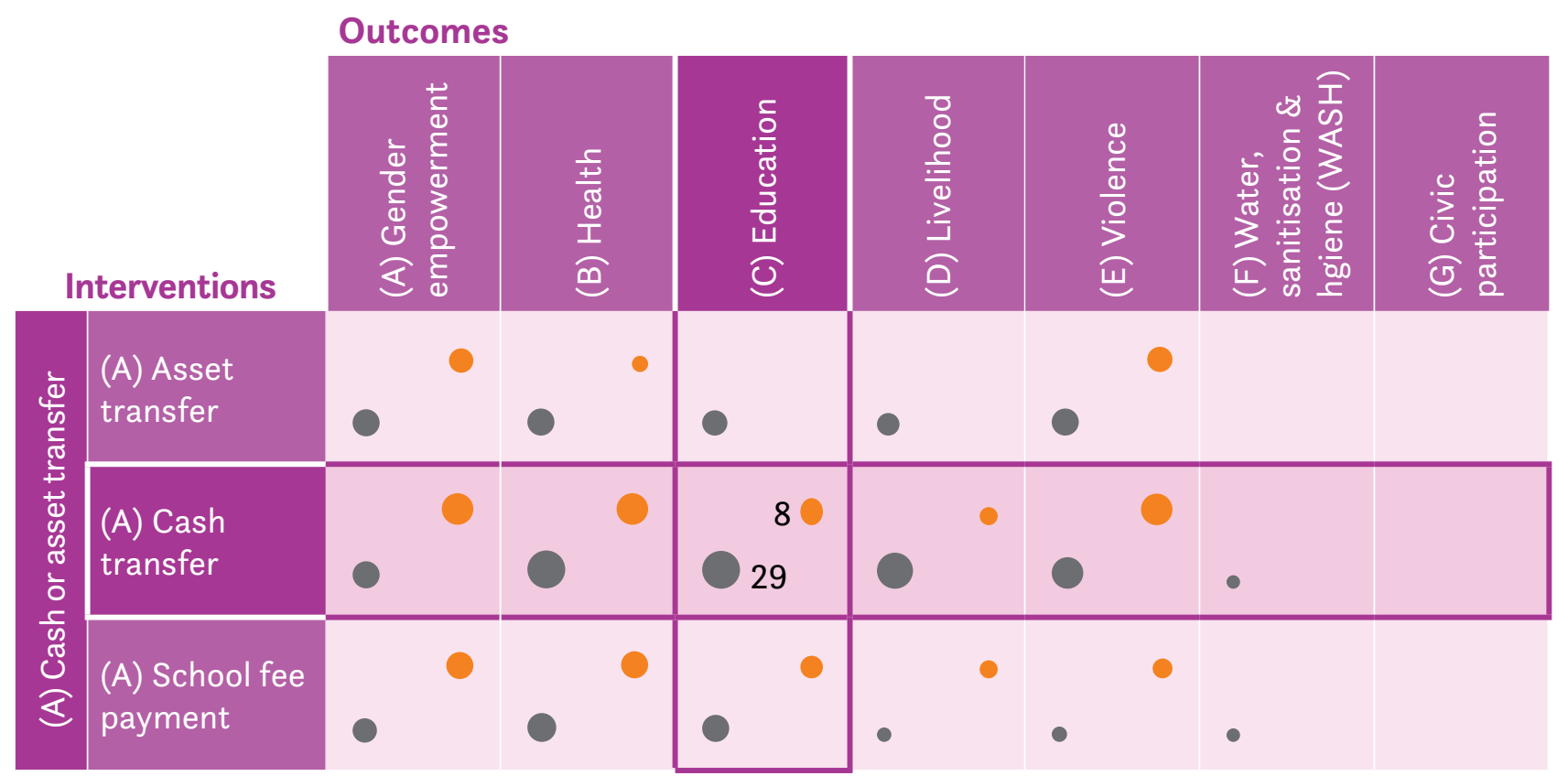

All types of evaluations All types of reviews 


\section{Livelihoods}

\section{RESULTS}

Outcomes related to girls' livelihoods include financial literacy, savings practices, preparation for paid work, and control and decision-making over the use of economic resources. Because work may interfere with school-going or indicate younger girls' risk, this category also includes measures such as reducing the amount of time or burden on girls to work for money or provide for household finances. Livelihoods and girl-specific economic effects of interventions accounted for a relatively small set of outcomes. Notably, several studies of large-scale social protection programs measured reductions in paid work to free girls' time for school and studying. Other intervention approaches, such as those offering savings accounts, vocational training, and financial literacy measured different outcomes, such as possessing savings, savings-related behaviors, and knowledge of budget-making.

The extract below portrays the concentration of review and evaluation evidence on the effects of financial literacy, sports, and tutoring interventions on adolescent girls' livelihoods: 6 reviews and 6 evaluations reported these results. These specialized interventions tend to be delivered in combination with other interventions, such as safe spaces and other types of girls' groups; 19 evaluations and 7 reviews reported the effects of girls' groups on livelihoods.

\section{EGM Extract 4: Livelihoods and Community-Based Services/Interventions}

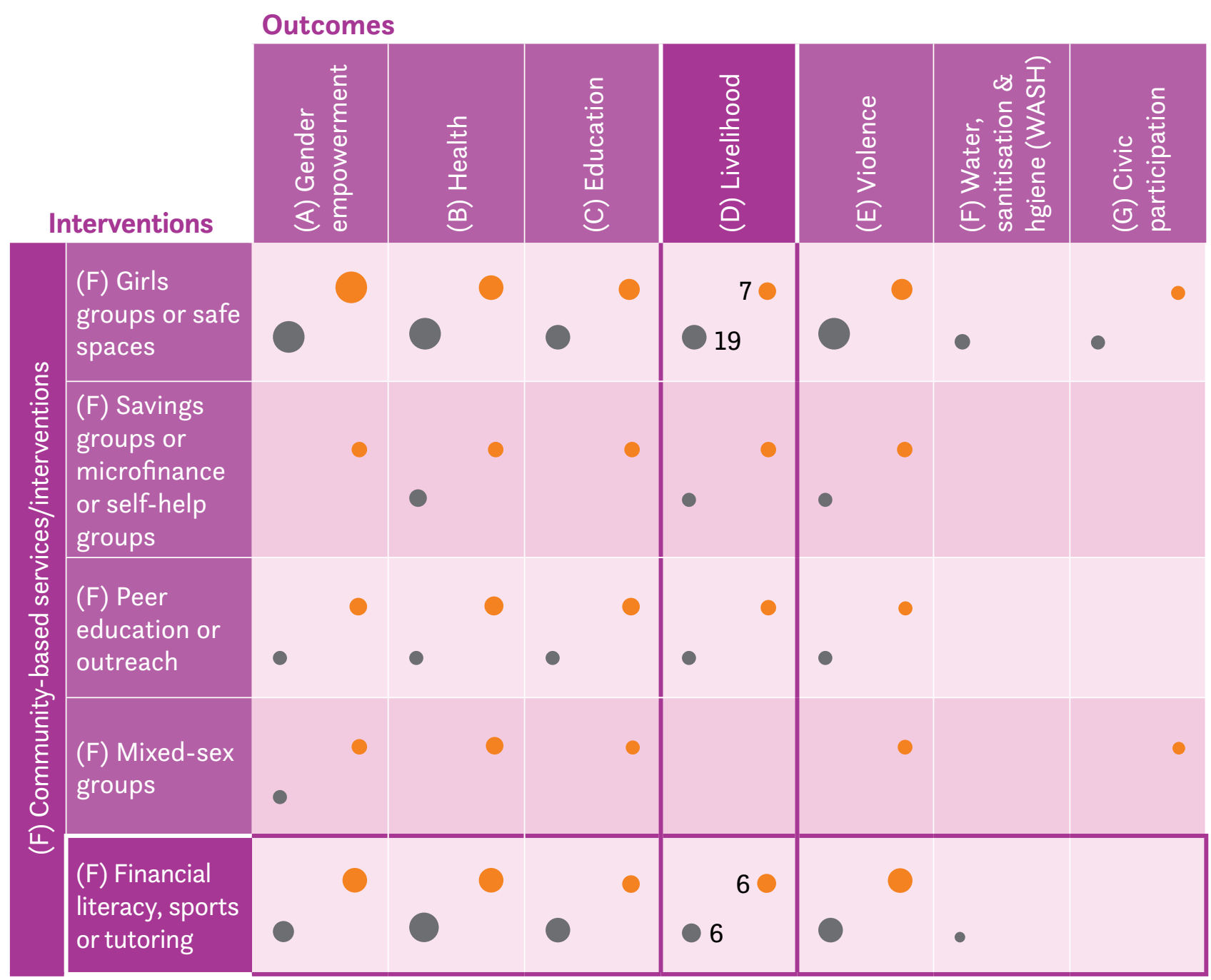




\section{Violence and Child Marriage}

\section{RESULTS}

Violence and child marriage outcomes typically center on girls' recognition and experience of various forms of violence, coercion, and abuse in public spaces, in school, and at the hands of an intimate partner including within their homes. Many studies draw on common definitions of violence from health surveys that describe sexual, physical, economic, and emotional violence. We include child marriage with these measures as it constitutes a violation of girls' bodily autonomy and human rights norms. Many interventions to promote girls' education aimed to delay marriage. Health education interventions and communitybased approaches also frequently measured outcomes related to child marriage and genderbased violence.

The following extract shows that safe spaces and other types of girls' groups are among the interventions with the most reported effects on violence and child marriage outcomes in reviews (14 reviews) and evaluations (29 evaluations). Alternatively, interventions such as legal and social protection are less studied for their effects on violence ( 5 evaluations and 7 reviews).

Case study 5: Girl Empower in Liberia

(Hallman et al., 2018; Ozler et al., 2020)

Quantitative design (cluster-RCT) including 13-14-year-old rural girls

In the Girl Empower program, girls' groups met for a mentorship program in which young, local female mentors facilitated weekly sessions using a 32-week life skills curriculum. The program aimed to equip girls with skills \& experiences so they could make healthy life choices \& avoid sexual violence. The additional impact of a cash transfer conditional on girl's attendance at meetings also was evaluated. The program decreased rates of child marriage $\&$ risky sexual behavior, effects that were sustained one-year post-program. The addition of the cash incentive to the mentorship program reduced the likelihood of marriage \& improved sexual behavior (\# sexual partners, abstinence, condom use) by around $50 \%$ more than the mentorship program alone. The program did not reduce sexual violence, though it influenced gender attitudes, which may contribute to reducing violence in the longer term. 
EGM Extract 5: Violence and Institution-Based Outside of Schools, and Community-Based Services/Interventions

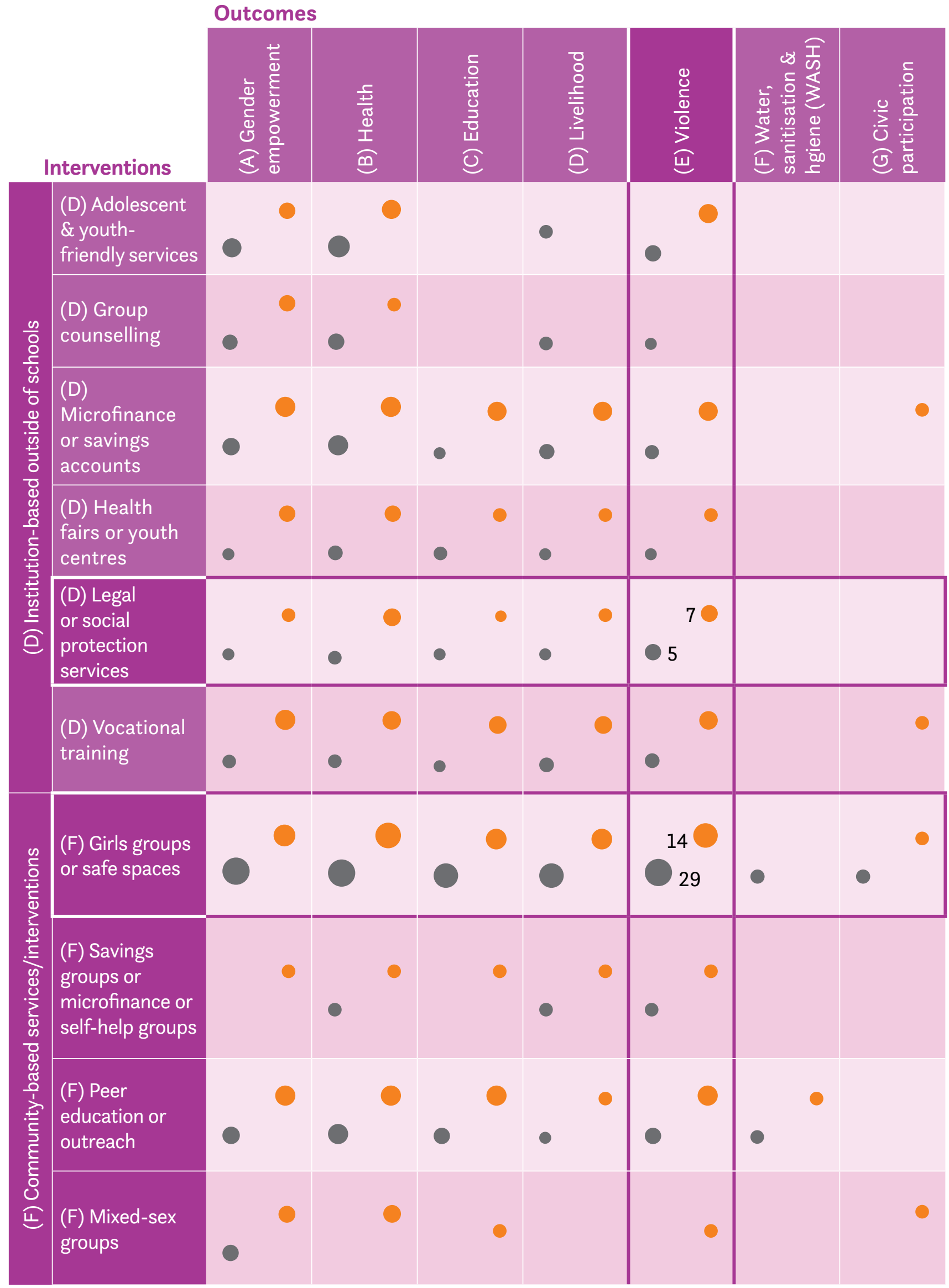

All types of evaluations All types of reviews 


\section{Water, Sanitation and Hygiene (WASH)}

\section{RESULTS}

WASH was the least-studied outcome domain in the review. This reflects that only a few studies focused on interventions in this domain, such as infrastructure upgrades to improve girls' access to clean water and/or sanitation facilities. Outcomes in this category include practices such as improved handwashing and MHM knowledge and practices.
While evidence on the effects of interventions on adolescent girls' WASH outcomes is limited, interventions that are relevant during crises have been evaluated. In particular, evaluations and reviews studied the effects of supply distribution including sanitary products, hygiene/dignity kits, and other forms of menstrual hygiene management supplies on girls' WASH outcomes. Effects were reported in 7 evaluation publications and 2 review publications as described in the extract below.

EGM Extract 6: WASH and Mass Information and Supply Distribution

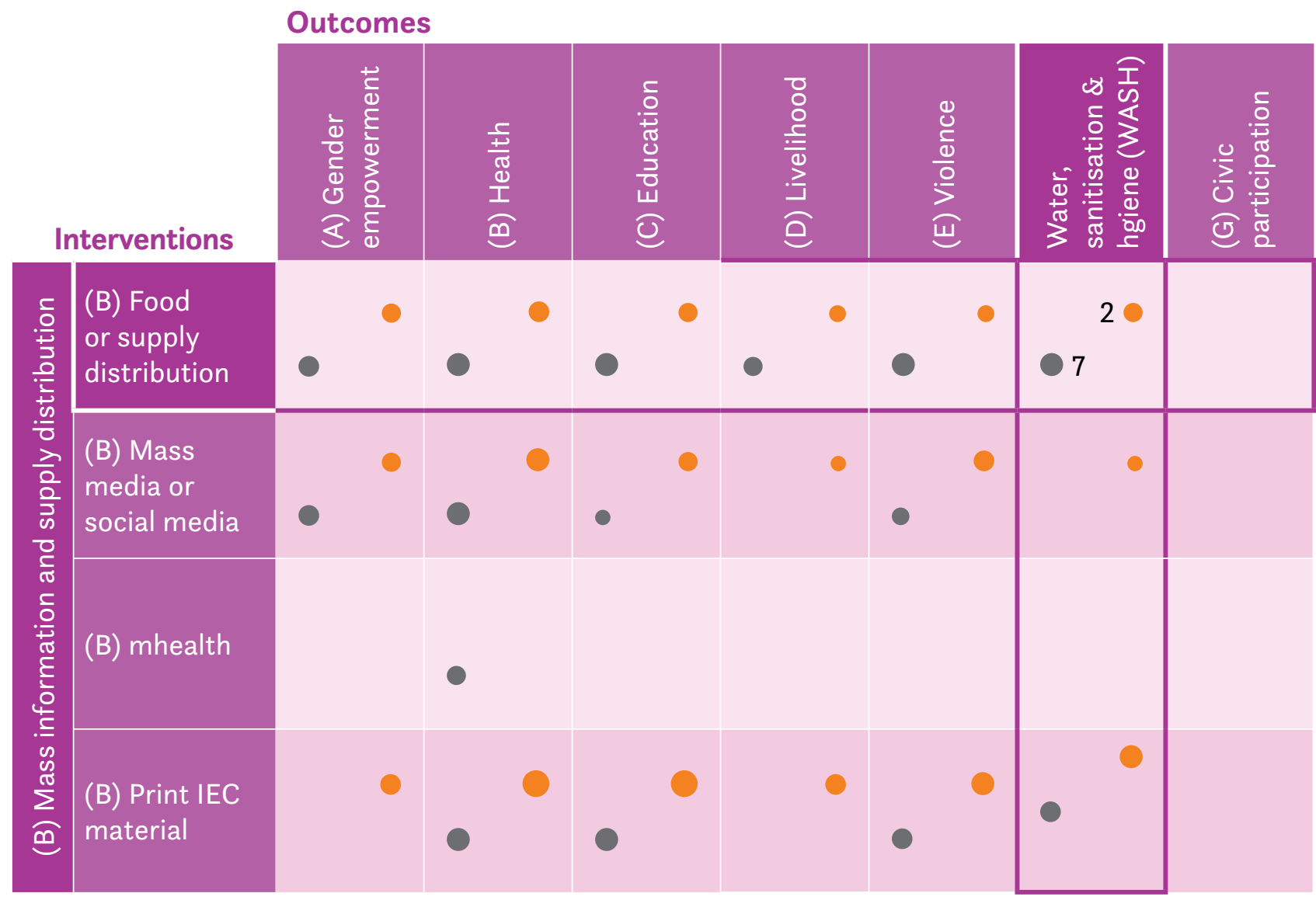

All types of evaluations All types of reviews 


\section{Other Outcomes}

\section{RESULTS}

A final category captured outcomes that were not easily grouped into the other domains. These include girls' participation in civic life and collective decision-making, an emerging theme that mirrors an expansion in the breadth of "life skills" and other forms of non-formal education that are offered in school and community-based interventions. The extract below confirms that this outcome domain had the least documentation of all the domains in our review. Few interventions were evaluated for their effects on adolescent girls' civic engagement and participation (e.g., girls' groups and safe spaces, mixed sex group activities and some vocational and health education trainings). Only two publications reported on girls' civic engagement and participation outcomes (a rigorous review and an evaluation study).

EGM Extract 7: Civic Participation and Community-Based Services/Interventions

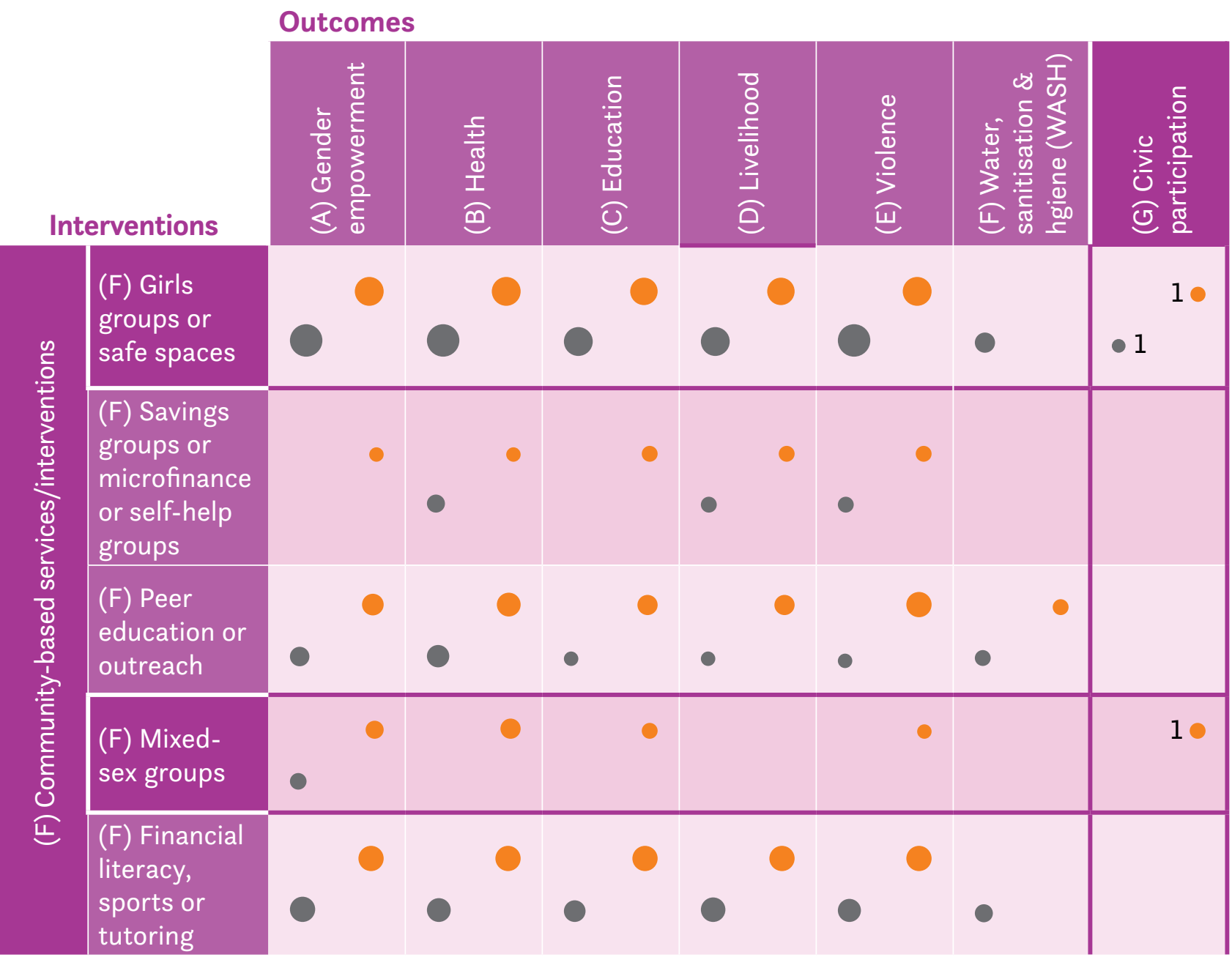

All types of evaluations All types of reviews

View the full Evidence Gap Map 


\section{Study Design Details}

Evaluation and review methods and the rigor of results varied substantially by intervention categories and subcategories. Experimental and quasi-experimental designs were common across intervention categories. Twenty quantitative or mixed-methods studies followed randomized controlled trial (RCT) experimental designs - often considered the "gold standard" in evaluation design - that enrolled at least 250 participants. Quasi-experimental studies and pilot RCTs included smaller intervention and control cohorts, which may be somewhat less rigorous than RCTs but common and often more feasible. 14 studies used either small-scale RCTs or quasi-experimental designs, or drew on secondary analysis of existing survey data to construct comparison groups. This final category also included studies that drew on real-world observational data, such as records on health service user profiles. While our analysis included grading studies based on their relative rigor, the Gap Map does not contain this information.

\begin{tabular}{|l|l|}
\hline Study Method & $\begin{array}{l}\text { Number of } \\
\text { Publications } \\
\text { (evaluation reports \& } \\
\text { reviews; total } n=171)\end{array}$ \\
\hline Quantitative & 105 \\
\hline Qualitative & 6 \\
\hline Mixed Methods & 28 \\
\hline Reviews & 32 \\
\hline
\end{tabular}

Different types of evaluation designs provided an important complement to the limited experimental data on interventions for adolescent girls. Large-scale secondary data analyses were applied for national or sub-national cash and asset transfer social protection programs. Most service delivery-related interventions, such as youth-friendly health services, were evaluated using observational methods collected at service delivery points, while evaluations of programs that combined facility upgrades or clinician trainings with broader multi-sectoral interventions used experimental methods. Social media or mass media approaches were typically assessed based on user data rather than data from pre-defined cohorts. A small set of studies captured qualitative data typically from mixed-methods evaluations. These reports provide valuable insights, such as participants' perspectives on programs goals, content, or implementation quality.

The number of evaluation and review publications that report effects of interventions within the seven intervention clusters is described below. The table reveals, for example, the highest concentration of evidence on the effects of community-based services, followed by schoolbased interventions.

\begin{tabular}{|l|l|l|}
\hline $\begin{array}{l}\text { Intervention } \\
\text { Clusters }\end{array}$ & $\begin{array}{l}\text { \# of } \\
\text { Evaluation } \\
\text { Reports }\end{array}$ & $\begin{array}{l}\text { \# of } \\
\text { Review } \\
\text { Reports }\end{array}$ \\
\hline $\begin{array}{l}\text { A. Cash or Asset } \\
\text { Transfer }\end{array}$ & 49 & 14 \\
\hline $\begin{array}{l}\text { B. Mass } \\
\text { information and } \\
\text { supply distribution }\end{array}$ & 24 & 8 \\
\hline $\begin{array}{l}\text { C. School-based or } \\
\text { formal educational } \\
\text { interventions }\end{array}$ & 44 & 21 \\
\hline $\begin{array}{l}\text { D. Institution- } \\
\text { based exclusive of } \\
\text { schools }\end{array}$ & 38 & 20 \\
\hline $\begin{array}{l}\text { E. Built } \\
\text { environment }\end{array}$ & 3 & 24 \\
\hline $\begin{array}{l}\text { F. Community- } \\
\text { based services/ } \\
\text { interventions }\end{array}$ & 55 & 38 \\
\hline $\begin{array}{l}\text { G. Complementary } \\
\text { interventions }\end{array}$ & 36 \\
\hline
\end{tabular}

Details on evaluation participants and contextual features do not appear in the Evidence Gap Map. However, these factors influence how programs are implemented and the likelihood of impact. Information on which types of girls were included in evaluations is particularly important because the same intervention can have varied effects on different subpopulations. Evaluations used diverse population 
descriptions and disaggregation approaches; no two studies evaluated effects for the same girl sub-populations. Most studies included broad descriptions of girl populations (such as girls 10-19 years). Some researchers stratified by age bands, and some evaluations deliberately engaged out of school and/or married girls.

Six studies included girls who were affected by conflict and residing in refugee or camps for Internally Displaced People (IDP). A small body of evidence described specific groups or segments of girls. For instance, evaluations of meanstested social protection programs included girls in households below a certain income threshold, while other classifications, such as orphans and vulnerable children, were also relatively common.

COVID-19-related restrictions and resulting economic instability present similar conditions to other economic, health, political, and environmental shocks, and are especially difficult for people living in crowded conditions. Thus, we included evaluations that accounted for humanitarian crises, such as complex emergencies; climate-related displacement and/ or economic or food insecurity, such as droughts and flooding; and health emergencies, such as the Ebola crises. We also noted contexts that included political crises or economic shocks that disrupt institutions and household economic status such as fiscal crises. With this broad definition, we found that a sizeable minority of studies (47) took place in contexts that qualified as "unstable." Not all evaluations that noted instability reported how such conditions might affect girls, nor the implications for intervention quality or feasibility of data collection.

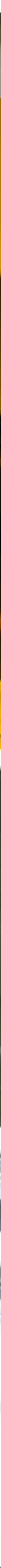




\section{Implications: Responding to Research Questions}

The findings of this review illustrate the amount of evidence available to inform decision-making on investments for adolescent girls. This information is relevant for investments in the evolving COVID-19 pandemic; the relevance also extends to global research. This section describes the implications in response to the four questions that shaped our review. These implications should be considered in future large-scale or localized crises resulting from infectious disease outbreaks or climate crises and widespread disruptions to economic activities, services, and schools.

1. Identify high-potential intervention approaches that contribute to reducing girls' risks of contracting infection (COVID-19), including interventions intended to promote girls' uptake of protective behaviors.

Surveys of adolescent girls and boys in lowresource contexts conducted since the onset of the pandemic find high levels of awareness of the disease and protective behaviors, along with extensive exposure to health education messages via mass media (Amin et al., 2020; Hamad et al., 2020; Jones et al., 2020; Matovu et al., 2021). By contrast, it is evident that structural factors such as crowded living conditions and poor water and sanitation access have hindered adolescent girls', as well as boys', practice of protective behaviors such as handwashing or physical distancing (Akmal, Hares, et al., 2020; Baird et al., n.d.; Hamad et al., 2020; Jones et al., 2020). Girls' participation in television, radio, and online learning are low and, in some settings, lagging, as the crisis continues. Factors like household wealth and age and gender disparities in access to mobile phones, can influence girls' participation (Amin et al., 2020; Bellerose et al., 2020; Jones et al., 2020; Youssef \& Jones, 2020).

Lessons from initiatives to distribute IEC materials, school supplies and study materials, and other tangible goods directly to girls offer relevant lessons. The pandemic necessitated rapid public education on COVID-19 control measures, such as handwashing and physical distancing. While some girls may have benefitted from bespoke messaging campaigns delivered by social media, unreliable phone access may undermine girls' access to vital information even when they are explicitly targeted. On a related note, evidence on the intra-household use of resources such as cash transfers illustrate the critical need to document girls' individual supply or resource needs and their preferences for resources. It also is vital to track whether and how resources distributed to households are shared among household members.

Lessons from intentional approaches to reach girls may inform tailored approaches that are relevant for girls' circumstances, thus increasing their accessibility. These insights are important for meeting pressing needs such as MHM supplies, which are among the goods that girls reported foregoing in the midst of economic crises early in the pandemic (Amin et al., 2020; Baird et al., n.d.; Hamad et al., 2020). Distributing MHM products, such as menstrual cups and pads, enables changes in girls' behaviors that economic barriers may otherwise prevent (Hennegan \& Montgomery, 2016; Kansiime et al., 2020; Oster \& Thornton, 2011). Evaluations of MHM supply interventions and/or puberty education materials are among the few that include documentation of girls' actual supply use, pointing to promising approaches for selecting and distributing products and providing information or written health education content.

A general conclusion from the available evidence is that to effectively reduce adolescent girls' direct infection risk, multiple approaches to health education must be combined with efforts to address structural factors.

The review revealed a concerning but unsurprising absence of evidence on interventions to address girls' immediate risks of COVID-19 transmission. Common features of emergency responses and health communication are rarely 
assessed for their varied effects on specific sub-populations' knowledge or ability to practice protective behaviors. Sex and age-disaggregated data on distribution, receipt, and control over information, resources, and/or cash that are intended to enable protective practices are essential to assess if girls benefit or additional approaches are required to reach them.

2. Identify short-term emergency interventions that hold promise to mitigate the secondary effects of COVID-19 on girls in contexts where recurrent outbreaks and control measures disrupt health and social services, schooling, or community-based programs.

Because closures associated with COVID-19 responses tend to substantially disrupt life, the intervention categories that involve relatively limited in-person contact and incorporate activities to meet critical needs may help mitigate the secondary effects of disruptions on adolescent girls. Concerningly, the review revealed major evidence gaps regarding interventions to mitigate the secondary effects of COVID-19. For example, interventions to address challenges exacerbated by the pandemic, including violence prevention and response, access to sexual and reproductive health services, and social isolation, stress and household conflict, are under-evaluated priorities. The pandemic has underscored the need to "learn by doing" programming in these vital areas to generate evidence on the quality and value of these approaches for girls in general, and for specific sub-populations, such as very young adolescents and those in marginalized communities.

Interventions in review Categories A and B may be particularly relevant in contexts with disrupted conditions. The implications of the available evidence on interventions in these categories follow.

\section{Category A: Cash and asset transfers, school fee payments}

Cash transfers, asset transfers, and provision of food subsidies often are used to address and offset the effects of acute economic shocks on households, including in the COVID-19 pandemic (Gentilini et al., 2020). Researchers have studied the effects of these approaches on adolescent girls' educational attainment, child marriage, and health in the medium to long-term. Despite the frequency of the use of transfers as social protection measures, we found little evidence on their effects on girls experiencing either a humanitarian emergency or household economic shocks, such as those related to COVID-19.

Responsive cash and asset transfers may contribute to offsetting risks resulting from household economic strains. For example, conditional and unconditional transfers can support girls to stay in school or remain unmarried. These, in turn, may immediately benefit girls in ways that have value in a crisis, for example, enabling girls to spend time studying rather than working or caring for a household. They may also reduce household economic strain, which may reduce pressures that jeopardize girls' schooling or independent learning activities. This is vital given that initial evidence which reveals that school closures and lockdown measures diverted girls' attention from school to caregiving and/or searches for paid work (Bellerose et al., 2020; Jones et al., 2020). Critical questions remain such as the level of transfers that is needed to influence behavior (the threshold effect) and whether transfers should be conditional, labeled, or unconditional.

The few evaluations that document the effects of cash or other types of transfers directly to adolescent girls, either on their own or alongside a caregiver, suggest that girls may use resources to meet their own needs and/or to benefit their households (Angeles et al., 2019; Baird et al., 2010, 2011, 2013; Handa et al., 2015). However, in situations where transfers are given to other household members, girls may not be able to control how they are used (Crea et al., 2015). There also is more to learn about the empowerment effects of cash and asset transfers.

The evidence illustrates the difficulty of targeting all intended beneficiaries with cash and asset transfers. Evidence is limited on measures to overcome administrative barriers and consistently reach targeted beneficiaries. However, a few promising approaches include a range of "cash plus care" approaches and targeted supports to improve coverage, such as deploying personnel to facilitate access to services and support schooling (Cluver et al., 2014; Hallfors et al., 2015). 


\section{Category B: Mass information and supply distribution}

Food and supply distribution, social and mass media, and IEC material distribution interventions to address acute needs are useful under disrupted conditions. However, evidence is scarce on the effects of these approaches for adolescent girls. Online "e-learning" and television schooling have been widely used as compensatory measures during school closures, although participation in these types of schooling measures has not been fully documented (UNICEF, 2020). The available evidence suggests that the combination of economic pressures and lost access to school and school-based programs present barriers for girls to access learning as well as nutrition (Akmal, Hares, et al., 2020; UNICEF, 2020). This is particularly concerning because spiking food prices, lost income, and loss of school nutrition programs appear to be an acute secondary effect of school closures and economic shutdowns for adolescent girls in low resource settings (Akmal, Crawfurd, et al., 2020; Amin et al., 2020; Banati et al., 2020; Bellerose et al., 2020; Hamad et al., 2020; Jones et al., 2020).

3. Identify longer-term developmental intervention approaches that hold promise for "building back better" with and for adolescent girls in the medium to long-term recovery period once disease transmission is controlled or stabilized globally but economic or social effects remain. These include measures that address long-term effects and those that relate to critical elements of physical or social infrastructure.

The bulk of evidence available on intervention effects for adolescent girls is relevant to mid- or long-term recovery contexts, when it is relatively feasible to rely on existing institutions such as schools or health facilities, or on community groups. Indeed, there is little distinction between approaches that may be valuable in addressing emerging challenges in the medium term, such as new or heightened barriers to girls' reenrollment or continuation in school once schools reopen, and approaches to address long-term development challenges.

At the time of writing, the widespread, enduring effects of COVID-19 had yet to be realized. For instance, varied national responses mean that school re-enrollment is ongoing in many places and data collection on schooling is underway. It is unclear how girls will experience the pandemic's lasting effects, or the aftermath of stresses related to school closures, social isolation, and household economic insecurity. Findings from several settings, such as Sierra Leone and Guinea post-Ebola, suggest that enduring mental health effects may have repercussions over time in the form of lost schooling and learning, household income, or social support (Bandiera et al., n.d.; W. C. Smith, 2020). Acute threats to household economic status may evolve into enduring conditions that threaten educational participation in particular, particularly among girls who previously had a tenuous hold on schooling (Akmal, Crawfurd, et al., 2020, p. 19; Bellerose et al., 2020; Hamad et al., 2020; Youssef \& Jones, 2020).

Mid- or long-term recovery periods provide an opportunity to integrate gender transformative approaches that help prepare girls and families alike to tackle social norms and practices and promote equity. These may include, for example, combinations of cash transfers, community mobilization, and direct school or communitybased activities with girls (Austrian \& Muthengi, 2013; A. Erulkar \& Medhin, 2014). Such combination approaches appear to be especially valuable in delaying marriage and promoting school-going for younger girls (Amin et al., 2016; A. Erulkar et al., 2017).

\section{Building back better with gender transformative approaches}

Efforts to reduce economic barriers or reduce household economic strain are likely to have pivotal value in the mid-term recovery period, including cash and asset transfers. For girls in households facing enduring economic impacts of the crisis, cash or school fee support may enable girls to continue or return to school or nonformal educational alternatives. The documented benefits of ongoing, predictable transfers are described above. One-off or short-term transfers' positive effects may wane over time if underlying economic conditions do not change (Alam et al., 2011; Millán et al., 2019). Evidence suggests that conditionalities that enable girls to continue school may have unintended consequences, including stress and mental health effects, if households view girls' school-going as a 

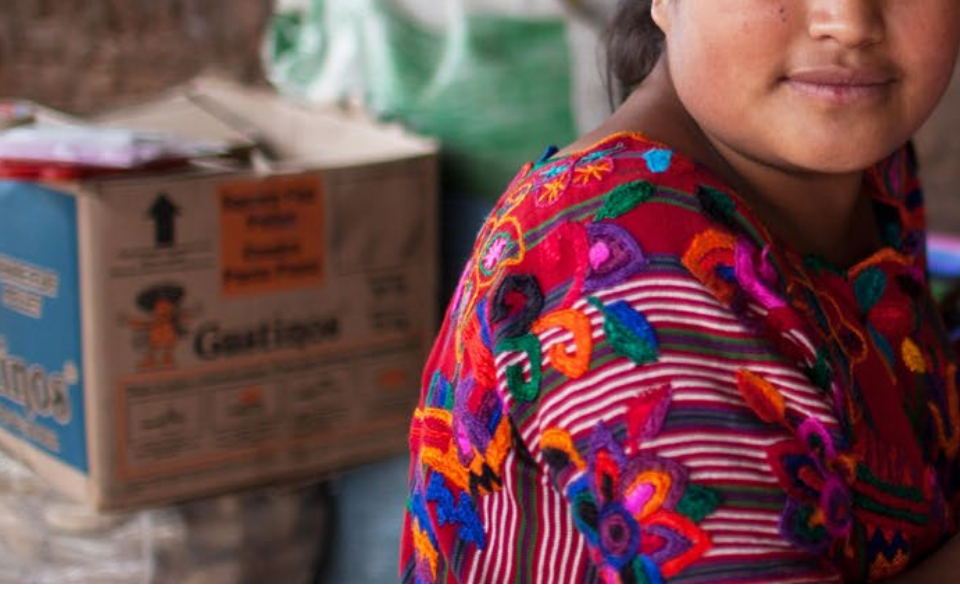

boost to household income (Baird et al., 2010). Tailoring cash and asset transfer interventions to girls' ages, and possibly pre-crisis school attainment, may help mitigate these risks. It also appears that other types of carefully-selected assets that respond to a perceived need in a given context may present a viable approach to tackling gendered and economic barriers to educational attainment, among other positive outcomes (Alam et al., 2011; Amin et al., 2016; A. Erulkar et al., 2017; A. Erulkar \& Medhin, 2014; Muralidharan \& Prakash, 2017).

\section{Interventions that incorporate emergency} measures, such as cash or asset transfers, that can be integrated into broader social protection systems have an important place in strategies to "build back better." Strengthening the civil infrastructure to enable such approaches, along with school and other institution-based approaches, could help counter the long-term economic effects of the pandemic that jeopardize families' ability to cover girls' schooling costs. Notably, structural interventions may pose new demands on girls to provide for other household needs. Tracking their impact to assess effects on girls of different ages in different contexts is vital to reduce this risk.

Tracking gender and age-specific effects of broader approaches to "build back better" may be an important aspect of making policies and programs gender transformative. For instance, gendered effects on school re-entry after COVID19-related closures may be subtle and slow to emerge. While families may express support for sending both girls and boys to school, gendered differences in social and economic support for schooling may lead to inequalities over time (Akmal et al., 2020; UNICEF, 2020). Evidence on cash transfers suggests that in some cases reducing the economic barriers to girls' education indirectly creates disparities that benefit boys as families reallocate resource savings to spend more on boys' education, such as by paying for private school fees (Ferreira et al., 2009; Lincove \& Parker, 2016; Muralidharan \& Prakash, 2017).

\section{Leveraging social infrastructure}

As situations evolve through an initial recovery, interventions that restore girls' access to services offer promise in the mid-term recovery period. Critical investments in recovery include services that can be delivered through existing social infrastructure, which can complement social protection measures such as transfers. Ensuring that social services respond to adolescent girls in all their diversity is a dimension of a gender-transformative vision for broader change. School-based health, gender, and violence prevention education; health services' responsiveness to adolescent girls; and protection and legal services are promising but understudied priorities. Similarly, dedicated action to reduce access barriers, such as referral mechanisms and support to register eligible families for social protection benefits or legal services, are promising but needing further research. Investment in "building back better" includes developing, adapting, and evaluating these approaches to achieve widespread coverage and quality at scale.

School-based health, gender rights awareness, and other educational measures offer important 
avenues to engage girls and their male peers in promoting girls' long-term health and well-being. Despite the promise of in-school health and life skills education, researchers find that as a result of restrictive policies on acceptable content and few resources for content development, the quality of teacher training and support and monitoring is inadequate (Aninanya et al., 2015; Arcand \& Wouabe, 2010). Recent reviews suggest that in settings with a heavy HIV burden, combining social protection measures such as cash transfers with other forms of support for families and girls' capabilities, or empowerment, have contributed to robust and enduring effects on reproductive health and preventing intimate partner violence (Denno et al., 2015; Peterman et al., 2017; Toska et al., 2016). More broadly, evidence indicates that short-term health-related benefits of interventions may fade over time (Duflo et al., 2015).

Promoting and protecting girls' health is critical to the recovery phase. Strengthening access to and quality of reproductive health services, and support for violence prevention or response, can address pressing needs that went unmet earlier in the pandemic. Enabling girls to protect themselves from HIV and/or early pregnancy remain particularly urgent challenges. Research results suggest that enhancing the quality, accessibility, and "youth-friendliness" of health services offers an important, comprehensive vision for service delivery. However, the concept does not translate consistently into a set of standards; as a result, planners may not use their scarce resources to prioritize aspects of "youth-friendliness" with the greatest potential for impact (Mazur et al., 2018). Integrating adolescents' perspectives into decision-making about service design is important given variability in needs by context and by group. However, adolescent engagement is rarely considered as part of a "youth-friendly" approach to health services (Mazur et al., 2018). Emerging findings suggest that tailoring interventions, for example by offering remote and static services, may increase service use among diverse adolescent populations (Smith et al., 2019).
Relatedly, physical infrastructure or "hardware" interventions are rarely studied for girl-level effects. The limited evidence available on the effects of these types of interventions on girls' centers on WASH interventions, especially MHM. Other under-studied physical infrastructure priorities that are relevant to adolescent girls are improving health and education service availability and quality. Studies highlight the lack of secondary schools, which pose a uniquely important barrier for progress in girls' education particularly in rural settings (Erulkar et al., 2017).

\section{Multi-level action including communities}

Interventions that work at multiple levels hold promise for improving a range of outcomes. These include combined household-level economic investments such as cash transfers and/or savings accounts; asset transfers; and/ or social interventions such as structured community dialogues on gender and girls' education (Haberland et al., 2018; Temin \& Heck, 2020). Such approaches tend to be delivered through communities rather than formal service delivery infrastructure that may not reach critical groups, such as married or out-of-school girls. As such, they offer flexibility for exploring responsive, innovative approaches.

Along with broad-ranging investments in services delivered through public sector institutions, existing evidence on community-based girls groups highlights the value and potential adaptability of such approaches during midterm recovery periods. Community-based girls' groups may offer an important and otherwise missing platform for girls to meet safely and restore valued relationships with mentors and peers (see Box 5.1). Where such programs were in place pre-crisis, restoring them is likely to be more feasible than developing new interventions. Limited evidence suggests that focusing on basic features of such programs, such as providing a meeting space and time, and delivering life skills content, may have important benefits for, for example, promoting self-esteem, or overcoming social isolation. (Haberland et al., 2018; Temin \& Heck, 2020). 


\section{1: Community-Based Girls' Groups in Humanitarian Settings and Economic Crises}

The design features and effects of communitybased girl groups in humanitarian contexts vary, as illustrated by the examples below.

- A multi-country experiment combined content for girls and parents/caregivers in a program in refugee camps with weak infrastructure, high insecurity, and common experiences of trauma. Evaluations found that the program had positive effects for girls and caregivers, but was insufficient to overcome structural barriers to reducing sexual violence against girls - its primary outcome. (Asghar et al., 2018; Stark, Seff, Asghar, et al., 2018a; Stark, Seff, Assezenew, et al., 2018b).

- An evaluation of girls' group interventions coincided with the Ebola crisis in West Africa (Bandiera et al., n.d., 2019) found that girls in communities with girls' clubs before outbreak-related suspensions were less likely to become pregnant over time than girls in other communities. The clubs offered information on topics such as sexual and reproductive health and time use options, which appeared to increase access to and use of contraceptive services, in turn enabling girls in participating villages to avoid pregnancy. However, the benefits did not extend to supporting girls to return to school and preventing violence or other common risks for girls during the Ebola crisis and reconstruction period.

- An evaluation of an intervention in postEbola Liberia found that a program that combined girls' groups and cash transfers had positive effects across multiple domains among girls who experienced high levels of violence (Hallman et al., 2018).

While evidence on girls' health and well-being in humanitarian settings is extremely limited, these mixed study results show the promise of community-based interventions in the mid-term recovery phase, when schools reopen but disruptions and social or economic instability persist(Noble et al., 2019).
4. Identify priority evidence gaps in areas such as: what kinds of interventions are evaluated; what outcomes are prioritized; which sub-populations of girls are included; and what forms of instability or exclusion are accounted for in intervention design or evaluations.

This review identified evidence to inform investments for adolescent girls under COVID19-related conditions at different stages of the pandemic. For instance, cash transfers and community-based girls' group programs appear to hold promise, while gender-transformative social protection mechanisms and education and health services, along with community or household-level action on harmful gender norms, also appear to benefit adolescent girls.

The review also highlighted many critical evidence gaps that limit policymakers' and implementers' ability to support adolescent girls as the COVID-19 pandemic evolves and, potentially, to avert the worst anticipated effects of the pandemic. Three broad observations on the state of the evidence emerged.

First, the lack of evidence on the effects on girls of common emergency protection measures, such as "dignity kits", is concerning. We did not find evaluation evidence on the reach and effects of household cash transfers, food and supply distribution, hotlines, and emergency health services on girls under 18 (see Box 5.2).

Second, assessing the relevance of evaluation results to different stages of the COVID-19 pandemic was undermined by insufficient documentation of contextual factors. This type of information is vital to interpreting evaluation results because it describes potential barriers to implementation that could have influenced intervention effects.

Third, adolescent girls are a heterogeneous sub-population; evaluation evidence confirms that approaches that have an impact on one group of girls may fail for others even in a single community (Amin et al., 2016; A. Erulkar, 2013; A. S. Erulkar \& Muthengi, 2009; Haberland et al., 2018). Despite this, evaluations rarely measured variable effects among different segments of adolescent girls, for example, 10 to 14 versus 15 to 19 year-olds. We excluded many evaluations

5 Basic supplies distributed to women and girls to help them maintain their dignity, e.g., menstrual supplies, soap, underwear, detergent, flashlight, toothpaste, toothbrush. 
of common interventions, such as school-based health education and HIV prevention approaches, because they did not disaggregate evaluation results by sex or age.

Despite an increasing number of rigorous reviews of individual studies, even the evidence on relatively common approaches remains fragmented. Nuanced findings that reflect adolescent girls' heterogeneity are often hard to disentangle from the unique features of individual study contexts and conditions, making the conclusions of our review preliminary.

\section{2: Absence of Evidence on Interventions and Outcomes Related to COVID-19}

The COVID-19 and Adolescent Girls framework identified a range of potential outcome domains salient to emergency, short-term effects of COVID-19 and longer-term, structural challenges that the pandemic has exposed or worsened. However, we found little to no evidence on effective approaches to address several outcome domains, as reflected in the gap map.

Two important outcome domains, technology for connectivity and transportation, returned no review results. While interventions made use of technologies such as mobile phones, and one study documented the effect of distributing bicycles on girls' school-going, we found no studies that explored questions related to girls' access to essential technologies, nor how to ensure girls' access to safe transportation.

COVID-19 specific concerns were largely absent from the evidence on relevant outcome domains, such as health promotion and violence prevention. Often, evidence on one outcome dominated the outcome domain: health tended to focus on sexual and reproductive health; violence and child marriage prevention on child marriage; and WASH on MHM. Outcomes with particular salience to ongoing pandemic outbreaks, such as access to and use of protective equipment, supplies or practices related to handwashing or the ability to practice social distancing, and the effectiveness of emergency services, such as hotlines and shelters, for girls experiencing household violence, did not appear in our searches.

Using the COVID-19 and Adolescent Girls Pathways as a guide, the paucity of evidence on interventions for adolescent girls indicates priorities for an adolescent girls and COVID-19 learning agenda. For instance, evidence is needed to inform measures to link girls with health, education, financial, and other services in the recovery phase. Another priority is to research effective strategies to contextualize multi-level approaches - as described above - to different settings, taking advantage of their relative flexibility. Implementation science that explores standards for program duration of community-based approaches, "dose" or participation per girl, and the effects of different "packages" of complementary content, such as financial literacy or civic participation activities, will help optimize these approaches and contribute to "building back better". 


\section{Summary Recommendations to Reduce COVID-19 Related Risk and Increase Opportunities for Adolescent Girls in Low- and Middle-Income Settings}

\section{To Reduce Immediate/Acute COVID-19}

\section{Infection Risks}

To effectively reduce adolescent girls' direct infection risk, multiple approaches to health education must be combined with efforts to address structural factors.

- Lessons from distribution of IEC materials, school supplies and study materials, MHM supplies, and other tangible goods directly to girls offer relevant lessons for delivery approaches for girls.

- Girls' uptake of vital protective practices is not guaranteed even if resources are distributed to households. Collecting sex and age-disaggregated data on distribution, receipt, and control over resources and/ or cash is vital to track whether and how household resources distributed are shared among household members.

\section{To Mitigate Secondary Effects}

Evidence is evolving regarding the types and duration of the pandemic's secondary effects. Relatedly, study results are limited to inform interventions to mitigate the secondary effects of COVID-19 on adolescent girls, such as school closures. Existing evidence indicates that interventions that rely on limited inperson contact and incorporate activities to meet girls' critical needs are feasible. Inclusive, gender responsive planning that balances the benefits of restrictions (e.g., on movement) with risks (e.g., girls' school dropout) is vital (UNICEF, 2021).

Emerging evidence on education during COVID-19-related school closures suggest that plans should focus on adolescent girls as schools open and close, offering different modalities that respond to girls' circumstances and needs. Students want active engagement with school staff to sustain learning, for example, safely exchanging materials with teachers (UNICEF, 2020).
Responsive cash and asset transfers can contribute to offsetting risks resulting from household economic strains. For example, transfers can support girls to stay in school or remain unmarried. It is essential to intentionally support and track coverage to ensure the intended beneficiary subpopulations are not overlooked, including directing targeted support measures to marginalized communities, households, and individuals.

The pandemic has underscored the need to prioritize "learning while doing" interventions to address urgent challenges exacerbated by the pandemic, including violence prevention and response, access to sexual and reproductive health services, and social isolation, stress and household conflict.

\section{To Build Back Better}

The opportunity to "build back better" postpandemic may offer a potential window to promote a gender-transformative vision for broader change. Ensuring that social services respond to adolescent girls in all their diversity is part of this vision. Tracking gender and age-specific effects of recovery approaches has potential to contribute to making policies and programs more gendertransformative.

- Interventions that incorporate stand-alone measures, such as cash or asset transfers, that can be integrated into comprehensive social protection systems have an important place in recovery strategies. "Cash-Plus" combination approaches appear to be especially valuable, while community-based girls' groups may offer an important and otherwise missing platform for girls to meet safely and restore valued relationships with mentors and peers. 
School-based health, gender, and violence prevention education; youth-friendly health services; and protection and legal services are promising intervention areas that are under-funded and under-studied. Tailoring interventions, for example by offering remote and static, clinic-based services, may increase service use among diverse subpopulations.

- Multilevel interventions that combine household-level economic investments, such as cash transfers and/or savings accounts, and/or social interventions such as structured community dialogues on gender and girls' education, also hold promise.

\section{To Fill Critical Evidence Gaps}

The review extracted evidence that suggests promising areas for investment at different stages of the pandemic, especially the mid- to long-term recovery. Overall, cash transfers and community-based girls' group programs hold promise for adolescent girls. Other potential investments include education and health services that are responsive to adolescent girls' particular circumstances. Community or householdlevel action to address harmful gender norms, for instance related to girls' education and GBV, also appear to benefit adolescent girls. Tailoring programming to girls' heterogeneity and layering multi-sectoral action are helpful approaches.

However, many critical evidence gaps limit policymakers' and implementers' ability to support adolescent girls as the COVID-19 pandemic evolves. The review turned up little to no evidence on effective approaches to address several outcome domains:
- Outcomes with particular salience to ongoing pandemic outbreaks, such as access to and use of protective equipment, supplies or practices related to handwashing social distancing did not appear in our searches.

- We found no evaluation evidence on the effects of common emergency measures on girls under 18 , including cash transfers, food and supply distribution, services for girls experiencing household violence, hotlines, and emergency health services.

- No studies explored questions related to girls' access to essential technologies, nor how to ensure girls' access to safe transportation.

In the recovery phase, more evidence is needed to inform programming to link girls with gender-transformative social protection, health, education, financial, and other services. This should include implementation science, which can increase understanding of the role of contextual factors. This evidence is vital to interpreting evaluation results and understanding what works for whom in what settings.

Nuanced findings that reflect adolescent girls' heterogeneity are needed to disentangle the unique features of individual beneficiaries, study contexts, and conditions. These findings should inform tailored recommendations that take account of the context and specific features of the targeted girl segment. 


\section{Annexes}

\section{Annex I: COVID-19 and Adolescent Girls Pathways: Summary of Hypothesized Risks and Opportunities (Illustrative)}

HEALTH THREAT: Disease pandemic w/out vaccine or cure; potential to overwhelm health services; novelty causes urgency; longer-term systemic health system threats.

\section{Risk to AGs}
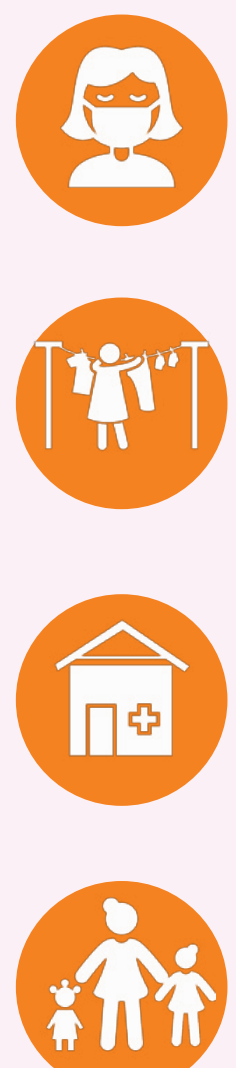

Long-term reliance on girls

as caregivers

Disruption of non-COVID health services \& products including $\mathrm{SRH}$ increases infective disease, suppresses demand for health services

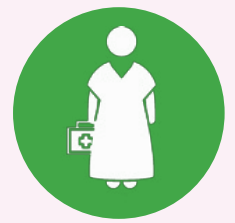

More investment in community health worker programs \& nonfacility-based primary/SRH care \& self-care

Extended limits on safe abortion 
EDUCATION THREAT: School closures; longer-term, dropouts \& decreased girls' enrollment.

Risk to AGs

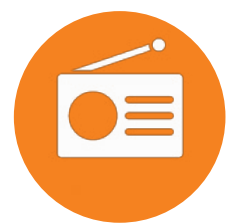

Poor quality of/limited access to alternative learning (radio, online).

\section{Girls lose social support of} school-mates.

Income \& gender disparity in access to alternative/online learning, tutoring, etc.
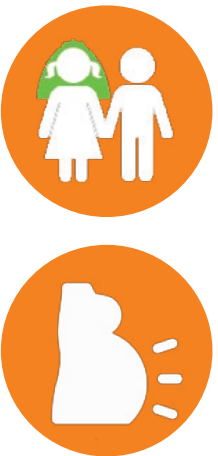

Increased early/unintended pregnancies \& exploitative relationships

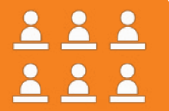

More child marriage in absence of schooling
Opportunities for AGs

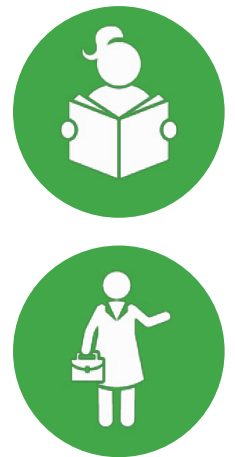

Tailor alternative learning to promote girls' participation

Community \& teacher/ principal education \& mobilization on girls' right to education, support to legal advocates

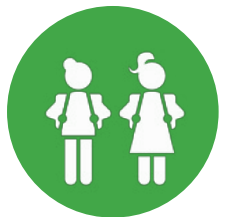

Informal schooling for CSE \& onramp to school; additional supports to continue girls' schooling including schooling stipends

LIVELIHOOD THREAT: Lockdowns cause business closures, unemployment; longer-term economic threat from unemployment, diversion of funds from social programs.

\section{Risk to AGs}
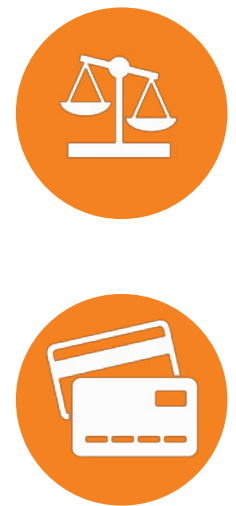

Exacerbates/reifies inequitable distribution of household reproductive labor, time poverty

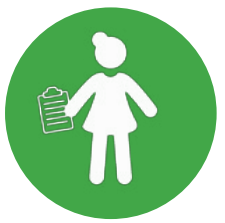

Girls as "everyone's credit card"; push into informal sector w/less security, more risks from work \& labor migration; less attention to long-term skills-building or training

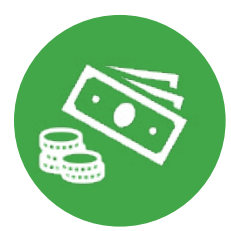

\section{Opportunities for AGs}

Jobs for young women as community preparedness agents; new cadres of community-based workers encompassed in public works programs

Girl group programming as platform for market-driven income generation

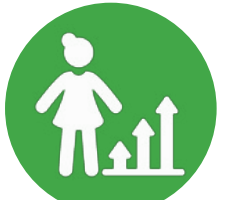

Community roles may improve perceived value of girls/ normative effects 
VIOLENCE THREAT: More violence especially at home; justice, medical, social services limited \& informal social support/sanctioning out of reach (differs for married girls).

\section{Risk to AGs}
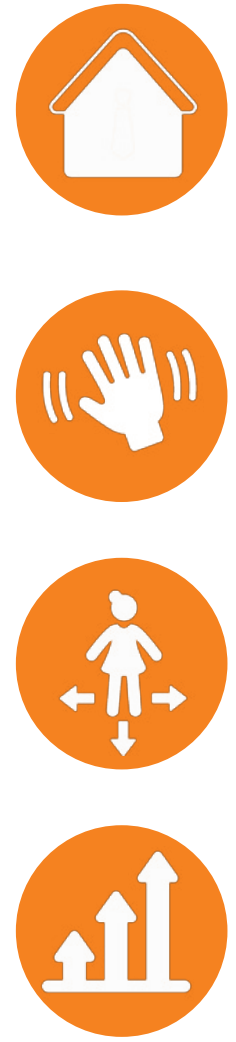

Travel restrictions, lockdowns, quarantines exacerbate risk of many forms of violence $\&$ perception, also fear of violence

Threats to safety, bodily integrity, mental health; fear of retribution/ reluctance to intervene w/out justice measures

Breakdown of social cohesion in communities leads to fear of community violence, restrictions on girls' movement $\&$ access to resources

Increase in harmful traditional practices due to family concerns re. shame, stigma in healthcare seeking, diversion of enforcement against FGM/C.

\section{Opportunities for AGs}

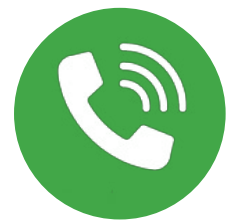

Increase access to violence hotlines for reporting, safe homes/shelters \& transportation; increase support for legal services; tailor SGBV survivor care to adolescent girls.

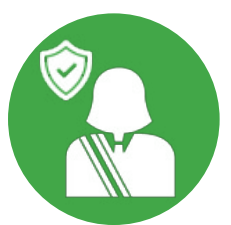

Girl-centered planning for safe access to community resources

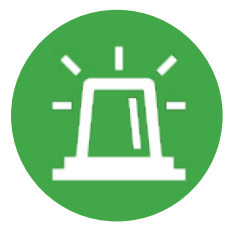

Development of community GBV response/ reporting/ support strategies

WATER AND SANITATION THREAT: Prevention emphasizes hand washing; lack of community resources, water shortages, inequitable access to supplies/resources.

Risk to AGs
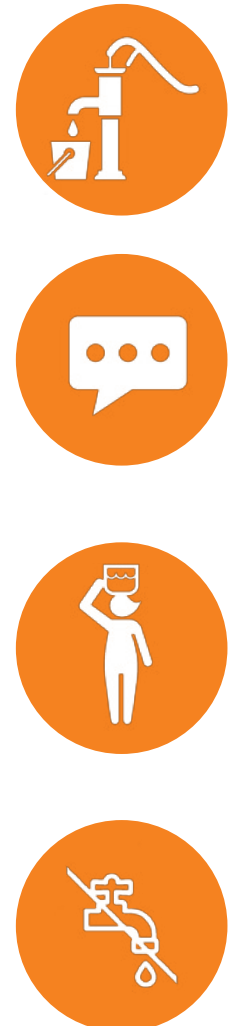

\section{Opportunities for AGs}

COVID infection risk in homes (esp. w/hygiene threats in caregiving) \& crowded water points

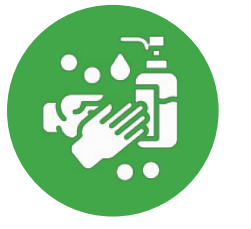

Girls/young women as handwashing educators \& promoters

Generic messaging fails to address girls'/other caregivers' increased water use needs, equipment distribution privileges male users \& excludes girls

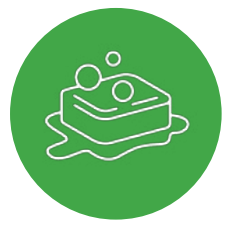

Equipment distribution targets girls as primary users

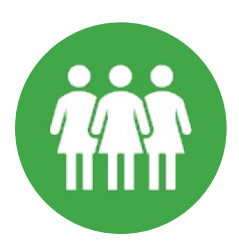

Women in leadership roles in community-based WASH infrastructure investments \& or training, employment in WASH management; encompass in public works programs

Demand on scarce water sources increases other health risks

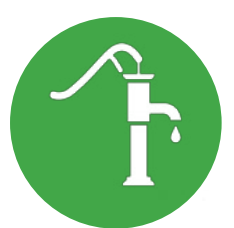

Increased commitment to safe, equitable access to community water sources; development of community mobilization strategies 
TRANSPORT THREAT: Lockdown limits transportation; longer-term public transportation disruption from economic effects, austerity measures.

\section{Risk to AGs}
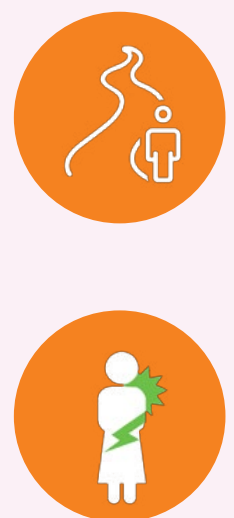

Travelling long, isolated distances using riskier-thanusual forms of transport increases sexual violence

Reduces married girls' recourse/ options in face of intimate partner violence

\section{Opportunities for AGs}

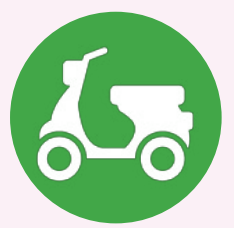

Incorporate provision of safe transportation into services for SGBV survivors

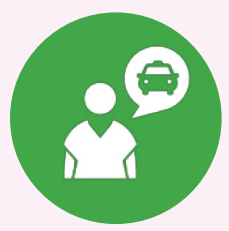

Investments in woman-managed transportation (e.g. safe rides $\&$ woman-run taxi companies) contribute to increased safe earning opportunities, enable young women to participate in training or employment further from home

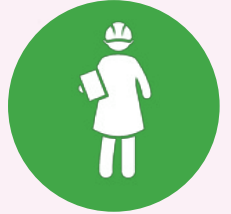

Gender \& age-responsive transportoriented public works employment targets young women

TECHNOLOGY THREAT: Digital connectivity is a lifeline under lockdown; digital gender \& context divides exacerbate inequalities.

\section{Risk to AGs}
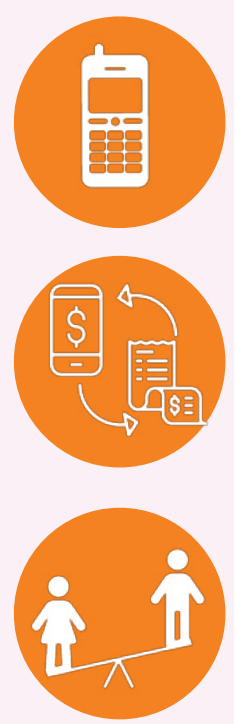

Girls are low in household power w/less mobile, internet access

High cost of data/airtime \& electricity increase girls' dependence on men for access

Increasing gender, SES, etc., inequity in info. access, social connections, electronic media skills

\section{Opportunities for AGs}

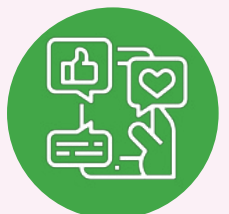

Innovation in interventions for critical media literacy, safe social media use interventions for adolescent girls.

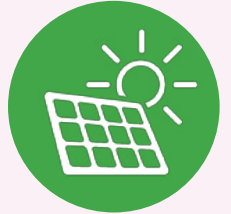

Emergence of tech. innovations, solar power sources, etc. for girls to leverage for empowerment \& development.

Increased importance of social media/technology during crisis as opening for engaging caregivers in new programs to support girls' access \& safe use of tech 


\section{ANNEX II: Evidence Review Methodology}

We conducted a structured literature review of major databases, including Google Scholar, PubMed, and JSTOR, which centered on a set of intervention approaches that engaged adolescent girls as a key population. Searches were limited to the period of 2005-2020.

\section{Databases and search terms}

\begin{tabular}{|l|l|}
\hline Databases: Google Scholar; JStor; PubMed & Search Terms: Adolescent girl* AND \\
\hline Nutrition assistance & Community mobilization \\
\hline Peer Education & mhealth \\
\hline Cash transfer & Mobile phone \\
\hline Child-friendly space & Adolescent and Youth-Friendly Services \\
\hline Menstrual hygiene management & Safe spaces \\
\hline Social protection & Social media intervention \\
\hline Asset transfer & Non-formal education \\
\hline Cash plus & Hand washing \\
\hline Comprehensive sexuality education & Health literacy intervention \\
\hline Supply distribution & Social media literacy \\
\hline Safe transportation & Livelihoods interventions \\
\hline Radio school & Positive youth development \\
\hline Girl-friendly & Social emotional learning \\
\hline Community health worker & Life skills \\
\hline Dignity kit & Livelihoods program \\
\hline Social insurance & \\
\hline
\end{tabular}

The scan generated an initial set of articles for title and abstract review. In addition to the systematic database search, we reviewed citations and reference lists from included studies and from commentaries and review articles that addressed adjacent topics, and hand-searched organizational websites to identify relevant grey literature that fit the criteria.

Based on the reviews, we further narrowed the studies to those that specified that adolescent girls were directly included in an evaluation. If other groups, such as young women or peerage boys, were also included, evaluations had to disaggregate findings by age and sex, and/ or incorporate gender and age effects into the analysis to be in the sample. Along with single evaluations, we also included systematic and other forms of structured literature reviews of evidence on interventions that reached adolescent girls and reported girl-specific results in their findings. We only included evaluations and reviews that described the research methods and sampling criteria. Both qualitative and quantitative or mixed methods evaluations were eligible for inclusion. 
Following the title and abstract review, we conducted a full-text review of the evaluations and reviews remaining in the sample. After the further review to verify fit with inclusion criteria and remove duplicate studies, we extracted details from the remaining studies. The extracted information covered:

Intervention components: organized by predefined categories and subcategories

- Methodology: Research method, rigor, sample size, and analytic approach and reported limitations

- Population: Description of girls included in study, including age and other sociodemographic information used in analysis; other populations studied

- Context: Region or regions, country, and community characteristics (rural, urban, peri-urban, camp), reference to emergency or instability, and type

Outcome domains: general areas studied.

Studies were divided into two categories by type:

1) reviews, which compile findings from a number of different studies; and 2) primary evaluations or assessments, which used a range of research methods to assess a single intervention. Some reviews were conducted around a given outcome, such as child marriage, and others gathered evidence on a given intervention type, such as youth-friendly reproductive health services, or serving a specific population, such as girls in urban settings. It was common for single evaluations to appear in multiple reviews. Primary evaluations or assessments included studies assessing a wide variety of interventions, from small-scale pilots to large experiments, as well as interventions supported by policies and implemented at scale. These studies may constitute "impact" evaluations, process evaluations, or they may address another feature of interventions' effects or implications for girls. In several cases, evidence on a single intervention appears across several articles or reports, so the number of evaluations and reviews included do not correspond with the number of discrete interventions described.

Population: We examined the demographic features reported for participants in the evaluations. Researchers used a wide variety of ways to describe their ages. For example, while several evaluations worked with girls' ages 10-14, and others used girls' ages 12-15 or 13-14. Along with evaluations that stratified by age, others focused on a single school grade level typically aligned with late primary school.

A few evaluations considered girls' exclusion or disadvantage, such as membership in an excluded ethnic group or past experience of trauma.

Studies in high HIV settings also targeted HIVpositive or HIV-negative girls, and/or selected only participants who were sexually active.

Other commonly excluded or overlooked groups, including girls living with disability, were either not specified as a target intervention group, or, in a few cases, specifically excluded.

Region: Studies were conducted across diverse low- and middle-income countries. A majority (100) of studies were conducted in sub-Saharan Africa. This was followed by 36 studies from Asia, which included 30 from South Asia. Five studies were from Latin America, and three from the Middle East/North Africa. In addition, 27 studies drew from interventions in multiple regions, either as reviews or from multi-region interventions.

Community Context: We took note of the community contexts of interventions where this information was reported. With the noteworthy exceptions of one review on humanitarian settings (Noble, French et al) and one review on urban contexts (Chant et al), most review articles included studies from multiple community types and contexts, often with minimal discussion of the role the context played. Much more detail was available among evaluations of single interventions. Rural settings were by far the most common sites for evaluations, with 77 studies specifying rural locations. Urban and periurban sites were included in fewer studies that accounted for a substantial set of interventions (51). A subset of these (24) included both urban and rural implementation sites. Refugee or internally displaced persons camps were the setting for six. Twelve studies were from interventions that were conducted or evaluated at district, province, or state-wide levels, and did not specify their specific community contexts, although these likely encompassed a variety of settings. 


\section{Rule Book: Detailed Categorization Process for Included Articles}

Conducted search using set terms (see Annex II); and hand searches $\rightarrow$ yielded 25,000+ results.

\section{Part 1: Screening for initial inclusion, exclusion}

Quantitative studies that met all criteria were selected for inclusion based on a PICOS framework ${ }^{6}$ and initial assessment of rigor:

Population $\rightarrow$ Specified adolescent girls (1019) in study population and disaggregated from other groups, e.g., boys, young women

Intervention $\rightarrow$

- Study aim included an assessment of any intervention implemented by an NGO or public sector agency that fit with predefined criteria

- Described: intervention content, scale, reach, time period

Comparison $\rightarrow$

- Study described criteria used to define randomized controls; non-random comparison under quasi-experimental approach; or process for non-comparison pre/post data collection design

Outcome $\rightarrow$ Hypothesized knowledge, attitudes, behaviors, and/or status changes among adolescent girls, across various outcome domains

- Study type $\rightarrow$ Experimental, quasiexperimental, and observational studies that clearly described method, sample, sampling criteria, and analysis

- Context $\rightarrow$ Low or middle-income country;

- published since 2005

Qualitative studies and review articles were assessed separately according to a modified set of criteria:

Population $\rightarrow$ Specified adolescent girls (10-19) and conducted data collection and analysis; for review articles, included a specific definition of "adolescent girls" as study population, and
Intervention $\rightarrow$

- Study aim included an assessment of an intervention or, in the case of systematic reviews, a group of interventions

- Described: intervention content, scale, reach, time period

Design $\rightarrow$

- Process evaluations, pilot evaluations

- Reviews (including systematic and non-systematic) to synthesize effects of interventions on adolescent girls on their own or as part of another sub-population (e.g., young people, women)

Evaluation $\rightarrow$ Of effects, perspectives, of interventions on adolescent girls' well-being

Research type $\rightarrow$

- Recognized qualitative methodology, such as in-depth interviews, focus group discussions;

- Systematic review or structured literature review

Context $\rightarrow$ Low or middle-income country;

- published since 2005

NOTE $\rightarrow$ Some publications only reported on the qualitative component of a mixed methods evaluation; in that case, the study outcomes are reported twice to account for qualitative and quantitative measurement of outcomes.

First narrowed to 815 results for full-text review based on population, intervention, methods, time period

Finalized to 171 based on full-text review based on all criteria

6 Population, Intervention, Comparison, Outcomes and Study (PICOS) framework often used to structure systematic reviews. For more information, see www.ncbi.nlm.nih.gov/pmc/articles/PMC4310146. 


\section{Part 2: Extraction and Organization of Study Components}

\section{Among studies that were selected for inclusion, we extracted the following basic descriptive information:}

\section{a. Intervention Categories and Subcategories:}

We defined intervention approaches according to categories of interventions that reflected what was likely to be feasible, appropriate, and common at various stages in the COVID-19 pandemic and recovery, reflecting judgments on: urgency of needs during a public health $\&$ economic crisis; feasibility under mobility restrictions; institutional ties and capacity; and relevance to adolescent girls.

Where studies involved interventions that included either multiple components within a single intervention package, or compared different intervention arms across domains, we indicated all domains of the intervention studied.

\begin{tabular}{|c|c|c|c|}
\hline Stage of crisis & $\begin{array}{l}\text { Intervention } \\
\text { category }\end{array}$ & Subcategory & Definition \\
\hline \multirow{3}{*}{$\begin{array}{l}\text { Highly } \\
\text { adaptable: } \\
\text { Emergency, } \\
\text { mitigation } \\
\text { \& recovery } \\
\text { phases }\end{array}$} & \multirow{3}{*}{$\begin{array}{l}\text { A. Cash or Asset } \\
\text { Transfer }\end{array}$} & Cash transfer & $\begin{array}{l}\text { Conditional, unconditional, or labeled transfers of money to } \\
\text { household or girl }\end{array}$ \\
\hline & & Asset transfer & $\begin{array}{l}\text { Conditional or unconditional transfers of valuable items with } \\
\text { defined monetary value }\end{array}$ \\
\hline & & $\begin{array}{l}\text { School fee } \\
\text { payment }\end{array}$ & $\begin{array}{l}\text { Payment of girls' school fees transferred from program to } \\
\text { school, bypassing household }\end{array}$ \\
\hline \multirow{4}{*}{$\begin{array}{l}\text { Emergency/ } \\
\text { crisis phase: } \\
\text { Responsive } \\
\text { to immediate } \\
\text { needs under } \\
\text { restricted } \\
\text { social contact } \\
\text { \& closures }\end{array}$} & \multirow{4}{*}{$\begin{array}{l}\text { B. Mass } \\
\text { information } \\
\text { and supply } \\
\text { distribution }\end{array}$} & $\begin{array}{l}\text { Food or supply } \\
\text { distribution }\end{array}$ & $\begin{array}{l}\text { Distribution of food stuffs or supplies, such as school supplies or } \\
\text { hygiene supplies to girl or to household with intent to reach girl }\end{array}$ \\
\hline & & $\begin{array}{l}\text { Mass media or } \\
\text { social media }\end{array}$ & $\begin{array}{l}\text { One-way or two-way messaging campaign intended to convey } \\
\text { important information or educational content to adolescent girls } \\
\text { via radio, television, social media, mobile phone messaging }\end{array}$ \\
\hline & & mHealth & $\begin{array}{l}\text { Referral or delivery of health services or counseling via mobile } \\
\text { phone, tablet, or computer }\end{array}$ \\
\hline & & $\begin{array}{l}\text { Print IEC material } \\
\text { distribution }\end{array}$ & $\begin{array}{l}\text { In-person or focused distribution of information, education, or } \\
\text { communications materials, books, or other print media to girls }\end{array}$ \\
\hline \multirow{9}{*}{$\begin{array}{l}\text { Mid-term } \\
\text { mitigation } \\
\text { to long-term } \\
\text { reconstruction }\end{array}$} & \multirow{6}{*}{$\begin{array}{l}\text { C. School- } \\
\text { based or formal } \\
\text { educational } \\
\text { interventions }\end{array}$} & $\begin{array}{l}\text { School feeding } \\
\text { or nutrition } \\
\text { supplementation }\end{array}$ & $\begin{array}{l}\text { School-based provision of meals or administration of } \\
\text { micronutrient supplementation }\end{array}$ \\
\hline & & $\begin{array}{l}\text { School-based } \\
\text { health or life skills } \\
\text { education }\end{array}$ & $\begin{array}{l}\text { Health or life skills curriculum delivered at school, by teachers or } \\
\text { other adult health educators, during or after school day; may be } \\
\text { for girls only or girls and boys together }\end{array}$ \\
\hline & & $\begin{array}{l}\text { School-based peer } \\
\text { education }\end{array}$ & $\begin{array}{l}\text { Health or life skills content delivered by peers during or after } \\
\text { school; minimal adult support }\end{array}$ \\
\hline & & Teacher trainings & $\begin{array}{l}\text { Specialized or intensive trainings in new or existing health or life } \\
\text { skills curriculum for teachers }\end{array}$ \\
\hline & & $\begin{array}{l}\text { School health clinics } \\
\text { or counseling }\end{array}$ & Health or social services provided on school grounds \\
\hline & & $\begin{array}{l}\text { School-based } \\
\text { outreach or } \\
\text { referrals }\end{array}$ & $\begin{array}{l}\text { Peer or professional outreach campaigns offered through school, } \\
\text { with informational content, or promotion/awareness building } \\
\text { for services via messaging campaigns, provide referrals to other } \\
\text { services }\end{array}$ \\
\hline & \multirow{3}{*}{$\begin{array}{l}\text { D. Institution- } \\
\text { based aside } \\
\text { from schools }\end{array}$} & $\begin{array}{l}\text { Adolescent and } \\
\text { youth-friendly } \\
\text { services }\end{array}$ & $\begin{array}{l}\text { Upgrades to service delivery infrastructure, supplies, referral } \\
\text { mechanisms, health worker visitations; and/or provider trainings } \\
\text { to improve youth-friendliness and promote young people's use } \\
\text { of services }\end{array}$ \\
\hline & & $\begin{array}{l}\text { Group counseling or } \\
\text { behavioral health }\end{array}$ & $\begin{array}{l}\text { Psychosocial interventions delivered by trained mental } \\
\text { health workers, including those recruited from community or } \\
\text { professionals }\end{array}$ \\
\hline & & $\begin{array}{l}\text { Micro grants, } \\
\text { microfinance or } \\
\text { savings accounts }\end{array}$ & $\begin{array}{l}\text { Provision of financial services through microfinance institutions, } \\
\text { mobile banking, or physical bank, with or without seed grants or } \\
\text { initial deposit }\end{array}$ \\
\hline
\end{tabular}




\begin{tabular}{|c|c|c|c|}
\hline Stage of crisis & $\begin{array}{l}\text { Intervention } \\
\text { category }\end{array}$ & Subcategory & Definition \\
\hline \multirow{13}{*}{$\begin{array}{l}\text { Mid-term } \\
\text { mitigation } \\
\text { to long-term } \\
\text { reconstruction }\end{array}$} & \multirow{3}{*}{$\begin{array}{l}\text { D. Institution- } \\
\text { based aside } \\
\text { from schools }\end{array}$} & $\begin{array}{l}\text { Health fairs or } \\
\text { youth centers }\end{array}$ & $\begin{array}{l}\text { One-time or recurrent public events offering health } \\
\text { educational information or platform for distributing } \\
\text { information; static or mobile centers offering general } \\
\text { engagement activities, such as games and computer access, } \\
\text { along with health education or information }\end{array}$ \\
\hline & & $\begin{array}{l}\text { Legal or social } \\
\text { protection } \\
\text { services or } \\
\text { referrals }\end{array}$ & $\begin{array}{l}\text { Referral mechanisms to existing social protection programs, } \\
\text { or links or establishment of legal services }\end{array}$ \\
\hline & & $\begin{array}{l}\text { Vocational } \\
\text { training }\end{array}$ & $\begin{array}{l}\text { Provision of structured vocational skills building to prepare } \\
\text { girls for employability or otherwise improve economic } \\
\text { opportunity }\end{array}$ \\
\hline & $\begin{array}{l}\text { E. Built } \\
\text { environment }\end{array}$ & $\begin{array}{l}\text { WASH } \\
\text { infrastructure }\end{array}$ & Upgrades to school or community WASH infrastructure \\
\hline & \multirow{5}{*}{$\begin{array}{l}\text { F. Community- } \\
\text { based } \\
\text { services/ } \\
\text { interventions }\end{array}$} & $\begin{array}{l}\text { Community- } \\
\text { based girls' } \\
\text { groups/safe } \\
\text { spaces }\end{array}$ & $\begin{array}{l}\text { Community mentor-led programming with life skills and } \\
\text { health content and recruitment from community; girls only }\end{array}$ \\
\hline & & $\begin{array}{l}\text { Community- } \\
\text { based savings } \\
\text { groups/ } \\
\text { microfinance or } \\
\text { self-help groups }\end{array}$ & Girl-only or intergenerational savings or lending groups \\
\hline & & $\begin{array}{l}\text { Financial literacy, } \\
\text { sports, or tutoring }\end{array}$ & $\begin{array}{l}\text { Specialized content on a topic or skill, delivered at community } \\
\text { level, with or without girls' groups }\end{array}$ \\
\hline & & $\begin{array}{l}\text { Peer education or } \\
\text { outreach }\end{array}$ & $\begin{array}{l}\text { Community-based peer education or use of peers in } \\
\text { disseminating health messages or conducting social } \\
\text { marketing for adolescent-specific products }\end{array}$ \\
\hline & & $\begin{array}{l}\text { Community- } \\
\text { based mixed-sex } \\
\text { groups }\end{array}$ & $\begin{array}{l}\text { Community-based groups offering health or life skills } \\
\text { programming to girls and boys at the same time }\end{array}$ \\
\hline & \multirow{4}{*}{$\begin{array}{l}\text { G. } \\
\text { Complementary } \\
\text { interventions }\end{array}$} & $\begin{array}{l}\text { Community based } \\
\text { boys' groups }\end{array}$ & $\begin{array}{l}\text { Boy-only community-based or school-based interventions } \\
\text { that operate in parallel to girls' interventions with structured } \\
\text { content and learning opportunities }\end{array}$ \\
\hline & & $\begin{array}{l}\text { Adult male } \\
\text { engagement }\end{array}$ & $\begin{array}{l}\text { Community-level engagement for husbands of adolescent } \\
\text { girls or adult men }\end{array}$ \\
\hline & & $\begin{array}{l}\text { Community } \\
\text { mobilization or } \\
\text { dialogues }\end{array}$ & $\begin{array}{l}\text { Structured or unstructured activities to promote girls' } \\
\text { participation in programming, address gender equity }\end{array}$ \\
\hline & & $\begin{array}{l}\text { Parent/caregiver } \\
\text { engagement }\end{array}$ & $\begin{array}{l}\text { Structured or unstructured activities directed specifically to } \\
\text { parents or non-parental caregivers of adolescents }\end{array}$ \\
\hline
\end{tabular}


b. Study Type: We organized sources according to the type of research methods used:

\begin{tabular}{|l|l|}
\hline Reviews & $\begin{array}{l}\text { Systematic review, meta- } \\
\text { analysis, review of reviews, } \\
\text { integrative review, etc. }\end{array}$ \\
\hline Quantitative & $\begin{array}{l}\text { RCT, experimental, quasi- } \\
\text { experimental, pre/post } \\
\text { comparison, analysis of large } \\
\text { data sets/artificial cohorts } \\
\text { (i.e., difference in difference } \\
\text { from census; DHS data) }\end{array}$ \\
\hline Qualitative & $\begin{array}{l}\text { Focus groups, in-depth } \\
\text { interviews, ethnography, } \\
\text { participatory exercises, } \\
\text { photovoice }\end{array}$ \\
\hline Mixed & $\begin{array}{l}\text { Authors specify and include } \\
\text { results on both qualitative } \\
\text { and quantitative methods }\end{array}$ \\
\hline
\end{tabular}

\section{c. Study Rigor:}

We did not systematically evaluate study rigor for qualitative or review articles.

Quantitative and mixed-methods studies were organized according to the rigor of their methodology, and grouped into three categories:

Q1. Highest rigor: Experimental, with random assignment to treatment arm (cluster or individual), discussion of sample size and

Q2. Quasi-Experimental or Observational; or experimental with lower rigor $\rightarrow$ Nonrandom assignment, but clear description of comparison groups or sites

Q3. Pre/post (low rigor) $\rightarrow$ Comparative data only between program participants at baseline and endline

\section{d. Description of population:}

Ages of girls included and description of age segmentation, if relevant

- Specified according to authors' groupings

- Additional socio-demographic descriptors reported for participants, such as marital status, school-going, disability, or migrant or refugee status

- Description of other populations included in intended beneficiary population, such as boys, young adult women, and/or assessment activities

\section{e. Context: We extracted information about the context for each study:}

Geographic region:

- Central, East, South Asia; Pacific

- Middle East/North Africa; East Africa, Southern Africa, West Africa;

- Latin America/Caribbean;

Name of country or countries where implemented

Description of community structural context:

- urban

- rural

- peri-urban

- refugee or internally displaced persons' camp

Inclusion of description of instability or crisis $\rightarrow$

- Whether instability or crisis of any kind was indicated; not defined; or not present

- Where instability was described:

$\rightarrow \quad$ Form or source of instability or crisis:

Infectious disease outbreak,

Complex humanitarian emergency,

Economic crisis,

Political crisis,

Climate-related crisis (drought, flooding) 
f. Domains for intended outcome(s) of intervention: Evaluations were categorized according to the domains of the intended girl-level outcomes that were measured based on a set of hypothesized "pathways," that roughly align with sectoral designations (i.e., education, health, violence prevention).

- Gender equitable social norms, voice, social connectedness, and other common measures of individual "empowerment" were included as a stand-alone category

- Child marriage was grouped with "violence,"
- No judgments were made based on type or level of findings - for example, knowledge, attitudes, behaviors, status changes related to education would all fall under "education" domain

Studies that assessed progress or change against multiple outcome domains were grouped for all relevant outcome categories. For example, where studies reported both girls' health and educational outcomes, they were assigned to both "health" and "education." 


\section{ANNEX III: References}

Abdullah, H. J., Ibrahim, A. F., \& King, J. (2010). Women's Voices, Work and Bodily Integrity in Pre-Conflict, Conflict and Post-Conflict Reconstruction Processes in Sierra Leone. IDS Bulletin, 41(2), 37-45.

Akmal, M., Crawfurd, L., Hares, S., \& Minardi, A. L. (2020). COVID-19 in Pakistan: A Phone Survey to Assess Education, Economic, and Health-Related Outcomes [Data set]. Harvard Dataverse.

https://doi.org/10.7910/DVN/EGQ4XO

Akmal, M., Hares, S., \& O’Donnell, M. (2020). Gendered Impacts of COVID-19 School Closures: Insights from Frontline Organizations.

https://www.cgdev.org/publication/gendered-impacts-covid-19-school-closures-insights-frontline-organizations

Alam, A., Baez, J. E., \& Del Carpio, X. V. (2011). Does cash for school influence young women's behavior in the longer term? Evidence from Pakistan. The World Bank.

Amin, S., Ahmed, J., Saha, J., Hossain, M., \& Haque, E. (2016). Delaying child marriage through community-based skills-development programs for girls: Results from a randomized controlled study in rural Bangladesh (p. 62). Population Council. https://www.popcouncil.org/uploads/pdfs/2016PGY_BALIKA_EndlineReport.pdf

Amin, S., Rob, U., Ainul, S., Hossain, M. I., \& Noor, F. R. (2020). Bangladesh: COVID-19 Knowledge, Attitudes, Practices \& Needs-Responses from three rounds of data collection among adolescent girls in districts with high rates of child marriage [COVID-19 Research \& Evaluations Brief]. Population Council.

https://doi.org/10.31899/pgy17.1013

Angeles, G., de Hoop, J., Handa, S., Kilburn, K., Milazzo, A., Peterman, A., \& Team, M. S. C. T. E. (2019). Government of Malawi's unconditional cash transfer improves youth mental health. Social Science \& Medicine, $225,108-119$.

Aninanya, G. A., Debpuur, C. Y., Awine, T., Williams, J. E., Hodgson, A., \& Howard, N. (2015). Effects of an Adolescent Sexual and Reproductive Health Intervention on Health Service Usage by Young People in Northern Ghana: A Community-Randomised Trial. PLOS ONE, 10(4), e0125267.

https://doi.org/10.1371/journal.pone.0125267

Arcand, J.-L., \& Wouabe, E. D. (2010). Teacher training and HIV/AIDS prevention in West Africa: Regression discontinuity design evidence from the Cameroon. Health Economics, 19 Suppl, 36-54.

https://doi.org/10.1002/hec.1643

Asghar, K., Mayevskaya, Y., Sommer, M., Razzaque, A., Laird, B., Khan, Y., Qureshi, S., Falb, K., \& Stark, L. (2018). Promoting Adolescent Girls' Well-Being in Pakistan: A Mixed-Methods Study of Change Over Time, Feasibility, and Acceptability, of the COMPASS Program. Prevention Science, 19(8), 1030-1042.

Austrian, K., \& Muthengi, E. (2013). "Safe and Smart Savings Products for Vulnerable Adolescent Girls in Kenya and Uganda: Evaluation Report.” Population Council.

Baird, S., Alheiwidi, S., Dutton, R., Mitu, K., Oakley, E., Woldehanna, T., \& Jones, N. (2020). Social Isolation and Disrupted Privacy Impacts of COVID-19 on Adolescent Girls in Humanitarian Contexts. Girlhood Studies, 13, 98-115. https://doi.org/10.3167/ghs.2020.130308

Baird, S., de Hoop, J., \& Özler, B. (2011). INCOME SHOCKS AND ADOLESCENT MENTAL HEALTH (Policy Research Working Paper No. 5644; p. 45). World Bank. https://openknowledge.worldbank.org/bitstream/ handle/10986/3407/WPS5644.pdf?sequence=1\&isAllowed=y

Baird, S., De Hoop, J., \& Özler, B. (2013). Income shocks and adolescent mental health. Journal of Human Resources, 48(2), 370-403.

Baird, S., McIntosh, C., \& Ozler, B. (2010). Cash or condition. Evidence from a Cash Transfer Experiment.

Baird, S., Seager, J., Sabarwal, S., Guglielmi, S., \& Sultan, M. (n.d.). Adolescence in the Time of COVID-19: 10.

Banati, P., Jones, N., \& Youssef, S. (2020). Intersecting Vulnerabilities: The Impacts of COVID-19 on the Psychoemotional Lives of Young People in Low- and Middle-Income Countries. The European Journal of Development Research, 32(5), 1613-1638.

https://doi.org/10.1057/s41287-020-00325-5

Bandiera, O., Buehren, N., Goldstein, M., Rasul, I., \& Smurra, A. (n.d.). Do School Closures During an Epidemic have Persistent Exects? Evidence from Sierra Leone in the Time of Ebola. 76. 
Bandiera, O., Buehren, N., Goldstein, M., Rasul, I., \& Smurra, A. (2019). The Economic Lives of Young Women in the Time of Ebola: Lessons from an Empowerment Program. World Bank, Washington, DC.

https://doi.org/10.1596/1813-9450-8760

Bellerose, M., Diaw, M., Pinchoff, J., Kangwana, B., \& Austrian, K. (2020). Pre-pandemic Influences on Kenyan Girls' Transitions to Adulthood during COVID-19. Girlhood Studies, 13(3), 133-150. https://doi.org/10.3167/ ghs.2020.130310

Cluver, L. D., Orkin, F. M., Boyes, M. E., \& Sherr, L. (2014). Cash plus care: Social protection cumulatively mitigates HIV-risk behaviour among adolescents in South Africa. Aids, 28, S389-S397.

Crea, T. M., Reynolds, A. D., Sinha, A., Eaton, J. W., Robertson, L. A., Mushati, P., Dumba, L., Mavise, G., Makoni, J. C., Schumacher, C. M., Nyamukapa, C. A., \& Gregson, S. (2015). Effects of cash transfers on Children's health and social protection in Sub-Saharan Africa: Differences in outcomes based on orphan status and household assets. BMC Public Health, 15 .

https://doi.org/10.1186/s12889-015-1857-4

Denno, D. M., Hoopes, A. J., \& Chandra-Mouli, V. (2015). Effective strategies to provide adolescent sexual and reproductive health services and to increase demand and community support. Journal of Adolescent Health, 56(1), $\mathrm{S}_{22-S} 41$.

Duflo, E., Dupas, P., \& Kremer, M. (2015). Education, HIV, and early fertility: Experimental evidence from Kenya. American Economic Review, 105(9), 2757-2797.

Erulkar, A. (2013). Evaluation of "Biruh Tesfa" (Bright Future) program for vulnerable girls in Ethiopia-Vulnerable Children and Youth Studies_Volume 8, Issue 2. Vulnerable Children and Youth Studies: An International Interdisciplinary Journal for Research, Policy and Care, 8(2), 182-192.

https://doi.org/10.1080/17450128.2012.736645

Erulkar, A., \& Medhin, G. (2014). Evaluation of health and education impacts of a girls' safe spaces program in Addis Ababa, Ethiopia.

Erulkar, A., Medhin, G., \& Weissman, E. (2017). The impact and cost of child marriage prevention in three African settings.

Erulkar, A. S., \& Muthengi, E. (2009). Evaluation of Berhane Hewan: A Program to Delay Child Marriage in Rural Ethiopia. International Perspectives on Sexual and Reproductive Health, 35(01), 006-014.

https://doi.org/10.1363/3500609

Ferreira, F. H. G., Filmer, D., \& Schady, N. (2009). Own and Sibling Effects of Conditional Cash Transfer Programs: Theory and Evidence from Cambodia $\mathbf{X}$ (Policy Research Working Paper No. 5001; Impact Evaluation Series No. 36 , p. 41). World Bank. http://documents1.worldbank.org/curated/en/341371468017440522/pdf/WPS5001.pdf

Gentilini, U., Almenfi, M., \& Dale, P. (2020). Social Protection and Jobs Responses to COVID-19: A RealTime Review of Country Measures (p. 422). World Bank. http://documents1.worldbank.org/curated/ en/448321588971503966/pdf/Social-Protection-and-Jobs-Responses-to-COVID-19-A-Real-Time-Review-ofCountry-Measures-May-8-2020.pdf

Haberland, N. A., McCarthy, K., \& Brady, M. (2018). A Systematic Review of Adolescent Girl Program Implementation in Low- and Middle-Income Countries: Evidence Gaps and Insights. Journal of Adolescent Health, 14.

Hallfors, D. D., Cho, H., Rusakaniko, S., Mapfumo, J., Iritani, B., Zhang, L., Luseno, W., \& Miller, T. (2015). The impact of school subsidies on HIV-related outcomes among adolescent female orphans. Journal of Adolescent Health, 56(1), 79-84.

Hallman, K., Guimond, M.-F., Özler, B., \& Kelvin, E. (2018). Girl Empower Impact Evaluation.

Hamad, S., Hamra, E. A., Diab, R., Hamad, B. A., Jones, N., \& Małachowska, A. (2020). Exploring the impacts of covid-19 on adolescents in the Gaza Strip (p. 12) [Policy Brief]. Gender and Adolescence: Global Evidence.

https://www.gage.odi.org/wp-content/uploads/2020/06/Exploring-the-impacts-of-covid-19-on-adolescents-inthe-Gaza-Strip.pdf

Handa, S., Peterman, A., Huang, C., Halpern, C., Pettifor, A., \& Thirumurthy, H. (2015). Impact of the Kenya Cash Transfer for Orphans and Vulnerable Children on early pregnancy and marriage of adolescent girls. Social Science \& Medicine, 141, 36-45. 
Hennegan, J., \& Montgomery, P. (2016). Do Menstrual Hygiene Management Interventions Improve Education and Psychosocial Outcomes for Women and Girls in Low and Middle Income Countries? A Systematic Review. PLOS ONE, 11(2), e0146985.

https://doi.org/10.1371/journal.pone.0146985

IPPF. (n.d.). COVID-19 pandemic cuts access to sexual and reproductive healthcare for women around the world | IPPF. Retrieved May 17, 2021, from https://www.ippf.org/news/covid-19-pandemic-cuts-access-sexual-andreproductive-healthcare-women-around-world

IRC \& VOICE. (n.d.). Where is the Money? How the Humanitarian System is Failing in its Commitments to End Violence Against Women and Girls. International Rescue Committee and VOICE.

https://www.rescue.org/sites/default/files/document/3854/whereisthemoneyfinalfinal.pdf

Jones, N., Gebeyehu, Y., Gezahegne, K., \& Tilahun, K. (2020). Exploring adolescents' experiences and priorities in Ethiopia under covid-19 [Policy Brief]. Gender and Adolescence: Global Evidence.

Kansiime, C., Hytti, L., Nalugya, R., Nakuya, K., Namirembe, P., Nakalema, S., Neema, S., Tanton, C., Alezuyo, C., Musoke, S. N., Torondel, B., Francis, S. C., Ross, D. A., Bonell, C., Seeley, J., \& Weiss, H. A. (2020). Menstrual health intervention and school attendance in Uganda (MENISCUS-2): A pilot intervention study. BMJ Open, 10(2), e031182. https://doi.org/10.1136/bmjopen-2019-031182

Lincove, J. A., \& Parker, A. (2016). The influence of conditional cash transfers on eligible children and their siblings. Education Economics, 24(4), 352-373. https://doi.org/10.1080/09645292.2015.1019431

Matovu, J. K. B., Kabwama, S. N., Ssekamatte, T., Ssenkusu, J., \& Wanyenze, R. K. (2021). COVID-19 Awareness, Adoption of COVID-19 Preventive Measures, and Effects of COVID-19 Lockdown Among Adolescent Boys and Young Men in Kampala, Uganda. Journal of Community Health. https://doi.org/10.1007/s10900-021-00961-w

Mazur, A., Brindis, C. D., \& Decker, M. J. (2018). Assessing youth-friendly sexual and reproductive health services: A systematic review. BMC Health Services Research, 18(1), 216.

Millán, T. M., Barham, T., Macours, K., Maluccio, J. A., \& Stampini, M. (2019). Long-term impacts of conditional cash transfers: Review of the evidence. The World Bank Research Observer, 34(1), 119-159.

Muralidharan, K., \& Prakash, N. (2017). Cycling to school: Increasing secondary school enrollment for girls in India. American Economic Journal: Applied Economics, 9(3), 321-350.

Noble, E., Ward, L., French, S., \& Falb, K. (2019). State of the evidence: A systematic review of approaches to reduce gender-based violence and support the empowerment of adolescent girls in humanitarian settings. Trauma, Violence, \& Abuse, 20(3), 428-434.

OECD. (2016). Donor Support to Southern Women's Rights Organisations. OECD DAC Network on Gender Equality (GENDERNET).

Oster, E., \& Thornton, R. (2011). Menstruation, Sanitary Products, and School Attendance: Evidence from a Randomized Evaluation. American Economic Journal: Applied Economics, 3(1), 91-100. $\underline{h}$ ttps://doi.org/10.1257/app.3.1.91

Peterman, A., Neijhoft, A., Cook, S., \& Palermo, T. M. (2017). Understanding the linkages between social safety nets and childhood violence: A review of the evidence from low-and middle-income countries. Health Policy and Planning, 32(7), 1049-1071.

Pinchoff, J., Santhya, K. G., White, C., Rampal, S., Acharya, R., \& Ngo, T. D. (2020). Gender specific differences in COVID-19 knowledge, behavior and health effects among adolescents and young adults in Uttar Pradesh and Bihar, India. PloS One, 15(12), e0244053. https://doi.org/10.1371/journal.pone.0244053

Smith, P., Tolla, T., Marcus, R., \& Bekker, L.-G. (2019). Mobile sexual health services for adolescents: Investigating the acceptability of youth-directed mobile clinic services in Cape Town, South Africa. BMC Health Services Research, 19(1), 584 .

Smith, W. C. (2020, August 5). Potential long-term consequences of school closures: Lessons from the 2013-2016 Ebola pandemic. Research Square.

https://doi.org/10.21203/rs.3.rs-51400/v1

Stark, L., Seff, I., Asghar, K., Roth, D., Bakamore, T., MacRae, M., D’Andon, C. F., \& Falb, K. L. (2018). Building caregivers' emotional, parental and social support skills to prevent violence against adolescent girls: Findings from a cluster randomised controlled trial in Democratic Republic of Congo. BMJ Global Health, 3(5). 
Stark, L., Seff, I., Assezenew, A., Eoomkham, J., Falb, K., \& Ssewamala, F. M. (2018). Effects of a social empowerment intervention on economic vulnerability for adolescent refugee girls in Ethiopia. Journal of Adolescent Health, 62(1), S15-S20.

Temin, M., \& Heck, C. J. (2020). Close to Home: Evidence on the Impact of Community-Based Girl Groups. Global Health: Science and Practice, 8(2), 300-324.

Toska, E., Gittings, L., Hodes, R., Cluver, L. D., Govender, K., Chademana, K. E., \& Gutiérrez, V. E. (2016). Resourcing resilience: Social protection for HIV prevention amongst children and adolescents in Eastern and Southern Africa. African Journal of AIDS Research, 15(2), 123-140.

UNFPA. (2020). Impact of the COVID-19 Pandemic on Family Planning and Ending Gender-based Violence, Female Genital Mutilation and Child Marriage [Interim Technical Note]. UNFPA. https://www.unfpa.org/sites/default/files/ resource-pdf/COVID-19_impact_brief_for_UNFPA_24_April_2020_1.pdf

UNICEF. (2020). COVID-19 and School Closures: Are children able to continue learning. UNICEF DATA. https:// data.unicef.org/resources/remote-learning-reachability-factsheet/

UNICEF. (2020). Guidance on Distance Learning Modalities to Reach All Children and Youth During School Closures. https://www.unicef.org/rosa/reports/guidance-distance-learning-modalities-reach-all-children-andyouth-during-school-closures

UNICEF. (2021). Reimagining Girls' Education. https://www.unicef.org/reports/reimagining-girls-education

van Zandvoort, K., Jarvis, C., Pearson, C. A. B., \& Davies, N. (2020). Response strategies for COVID-19 epidemics in African settings: A mathematical modelling study. London School of Hygiene and Tropical Medicine.

https://cmmid.github.io/topics/covid19/covid-response-strategies-africa.html

Youssef, S., \& Jones, N. (2020). Double crisis: Effects of a pandemic and economic crisis on Lebanon's most vulnerable adolescents (p. 11) [Policy Brief]. 


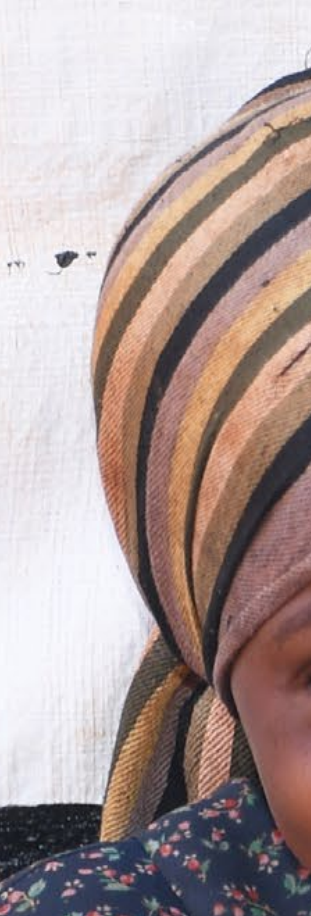

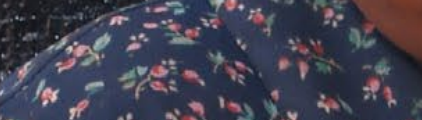

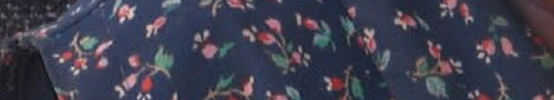
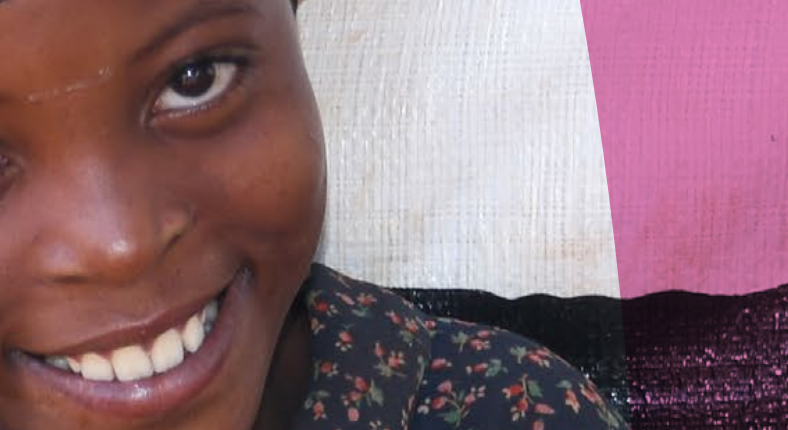

कo

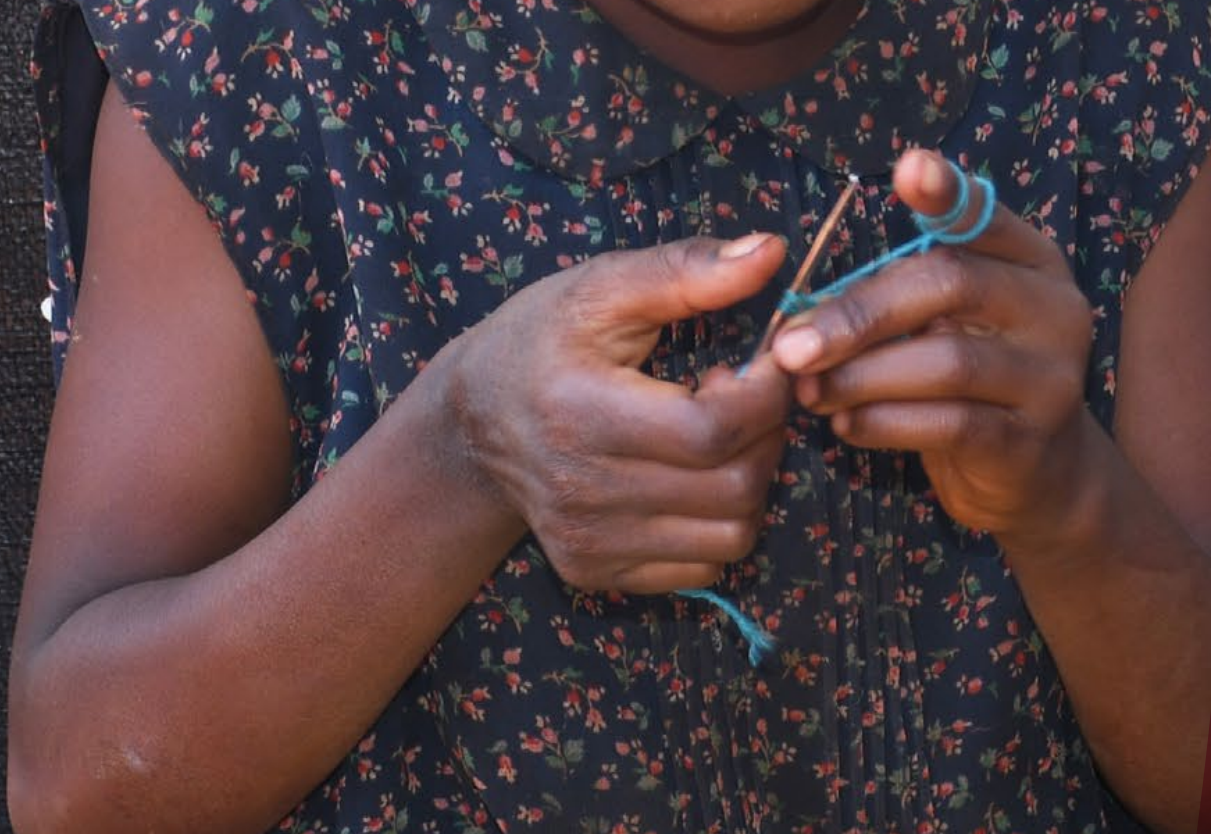

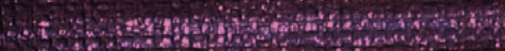

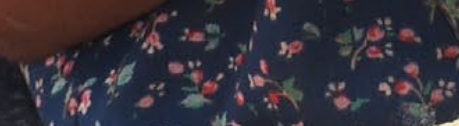

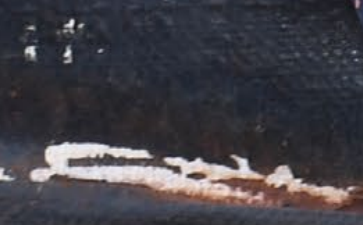

(1)

(it $x=$ nitis

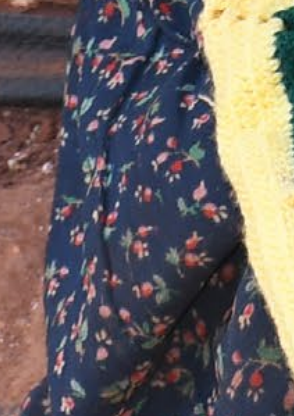

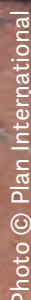
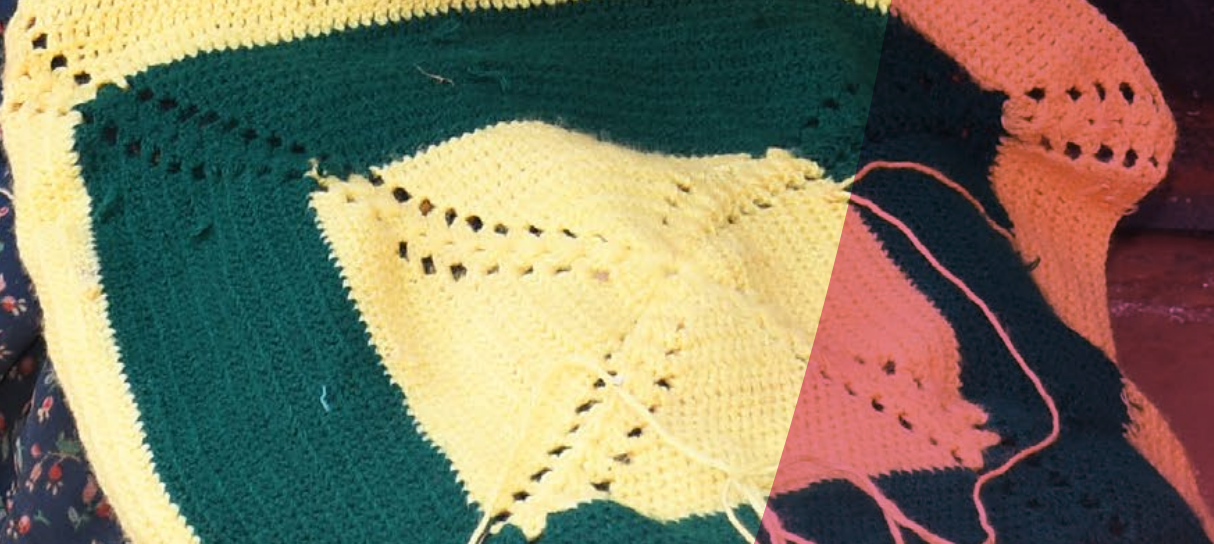
